\title{
Non-compact duality, super-Weyl invariance and effective actions
}

\section{Sergei M. Kuzenko}

Department of Physics M013, The University of Western Australia, 35 Stirling Highway, Perth, W.A. 6009, Australia

E-mail: sergei.kuzenko@uwa.edu.au

ABSTRACT: In both $\mathcal{N}=1$ and $\mathcal{N}=2$ supersymmetry, it is known that $\operatorname{Sp}(2 n, \mathbb{R})$ is the maximal duality group of $n$ vector multiplets coupled to chiral scalar multiplets $\tau(x, \theta)$ that parametrise the Hermitian symmetric space $\operatorname{Sp}(2 n, \mathbb{R}) / \mathrm{U}(n)$. If the coupling to $\tau$ is introduced for $n$ superconformal gauge multiplets in a supergravity background, the action is also invariant under super-Weyl transformations. Computing the path integral over the gauge prepotentials in curved superspace leads to an effective action $\Gamma[\tau, \bar{\tau}]$ with the following properties: (i) its logarithmically divergent part is invariant under super-Weyl and rigid $\mathrm{Sp}(2 n, \mathbb{R})$ transformations; (ii) the super-Weyl transformations are anomalous upon renormalisation. In this paper we describe the $\mathcal{N}=1$ and $\mathcal{N}=2$ locally supersymmetric "induced actions" which determine the logarithmically divergent parts of the corresponding effective actions. In the $\mathcal{N}=1$ case, superfield heat kernel techniques are used to compute the induced action of a single vector multiplet $(n=1)$ coupled to a chiral dilaton-axion multiplet. We also describe the general structure of $\mathcal{N}=1$ super-Weyl anomalies that contain weight-zero chiral scalar multiplets $\Phi^{I}$ taking values in a Kähler manifold. Explicit anomaly calculations are carried out in the $n=1$ case.

KEYwords: Supergravity Models, Superspaces, Supersymmetric Effective Theories, Supersymmetry and Duality

ARXIV EPRINT: 2006.00966 


\section{Contents}

1 Introduction 1

2 Superconformal higher-derivative actions 3

2.1 Local $\mathcal{N}=1$ supersymmetry 4

2.2 Local $\mathcal{N}=2$ supersymmetry 5

2.3 Relating the $\mathcal{N}=2$ and $\mathcal{N}=1$ actions 6

3 Super-Weyl anomalies $\quad 7$

$\begin{array}{ll}3.1 \mathcal{N}=1 \text { super-Weyl anomalies } & 7\end{array}$

$\begin{array}{lll}3.2 \mathcal{N}=2 \text { super-Weyl anomalies } & 10\end{array}$

4 Quantisation $\quad 10$

5 Heat kernel calculations (I) $\quad \mathbf{1 3}$

$\begin{array}{lll}5.1 & \text { Generalised Schwinger-DeWitt representation } & 13\end{array}$

$\begin{array}{ll}5.2 \text { Evaluation of the heat kernel in flat superspace } & 14\end{array}$

$\begin{array}{ll}5.3 \text { Super-Weyl anomaly } & 16\end{array}$

$6 \quad$ Heat kernel calculations (II) 16

6.1 Generalised Schwinger-DeWitt representation $\quad 17$

$\begin{array}{ll}\text { 6.2 Chiral and super-Weyl anomalies } & 20\end{array}$

$\begin{array}{lll}7 & \text { Concluding comments } & 21\end{array}$

$\begin{array}{ll}\text { A Super-Weyl transformations } & 22\end{array}$

\section{Introduction}

It is well known that the group of electromagnetic duality rotations of free Maxwell's equations is the compact group $\mathrm{U}(1)$, assuming the duality invariance of the energymomentum tensor. Almost forty years ago, it was shown by Gaillard and Zumino [1, 2] that the non-compact group $\operatorname{Sp}(2 n, \mathbb{R})$ is the maximal duality group of $n$ vector field strengths $F_{a b}^{I}=-F_{b a}^{I}$ in the presence of scalar $\tau^{i}$ parametrising the homogeneous space $\mathrm{Sp}(2 n, \mathbb{R}) / \mathrm{U}(n) .{ }^{1}$ In the absence of scalars, the largest duality group proves to be $\mathrm{U}(n)$, the maximal compact subgroup of $\operatorname{Sp}(2 n, \mathbb{R})$. These results admit a natural extension to the case when the pure vector field part $L(F)$ of the Lagrangian $L(F, \tau)$ is a nonlinear self-dual theory $[7-11]$ (see $[6,12,13]$ for reviews), for instance the Born-Infeld theory. However, in

\footnotetext{
${ }^{1}$ The Gaillard-Zumino approach was inspired by patterns of duality in extended supergravity theories [35], see [6] for a review.
} 
the case that $L(F)$ is quadratic, the $F$-dependent part of $L(F, \tau)$ is also invariant under the Weyl transformations in curved space. Then, computing the path integral over the gauge fields leads to an effective action, $\Gamma[\tau]$, such that its logarithmically divergent part is invariant under Weyl and rigid $\operatorname{Sp}(2 n, \mathbb{R})$ transformations, see, e.g., [14, 15] for formal arguments. Both symmetries are anomalous at the quantum level, but the logarithmically divergent part of the one-loop effective action is invariant under these transformations. This simple observation offers a powerful tool to construct higher-derivative actions with required symmetry properties as induced actions. For instance, this idea was employed in an important paper by Buchbinder, Pletnev and Tseytlin [16] to derive the bosonic sector of $\mathcal{N}=4$ conformal supergravity. ${ }^{2}$

The analysis of ref. [16] was based in part on the earlier results of Osborn [19] who studied the effective action, $\Gamma[\tau, \bar{\tau}]$, obtained by integrating out the quantum gauge field in the model with classical action

$$
L(F, \tau, \bar{\tau})=-\frac{1}{4} \mathrm{e}^{-\varphi} F^{a b} F_{a b}+\frac{1}{4} \mathfrak{a} \tilde{F}^{a b} F_{a b}, \quad \tau=\mathfrak{a}+\mathrm{i} e^{-\varphi} .
$$

Here $\tilde{F}^{a b}$ is the Hodge dual of $F_{a b}$, and $\mathfrak{a}$ and $\varphi$ are the axion and dilation, respectively. The logarithmic divergence of the effective was shown to have the form

$$
\begin{aligned}
\mathfrak{L}= & \frac{1}{2(\operatorname{Im} \tau)^{2}}\left[\mathcal{D}^{2} \tau \mathcal{D}^{2} \bar{\tau}-2\left(R^{a b}-\frac{1}{3} \eta^{a b} R\right) \nabla_{a} \tau \nabla_{b} \bar{\tau}\right] \\
& +\frac{1}{12(\operatorname{Im} \tau)^{4}}\left[\alpha \nabla^{a} \tau \nabla_{a} \tau \nabla^{b} \bar{\tau} \nabla_{b} \bar{\tau}+\beta \nabla^{a} \tau \nabla_{a} \bar{\tau} \nabla^{b} \tau \nabla_{b} \bar{\tau}\right]
\end{aligned}
$$

where

$$
\mathcal{D}^{2} \tau:=\nabla^{a} \nabla_{a} \tau+\frac{\mathrm{i}}{\operatorname{Im} \tau} \nabla^{a} \tau \nabla_{a} \tau,
$$

and $\alpha$ and $\beta$ are numerical parameters. The Lagrangian (1.2) is manifestly invariant under $\mathrm{SL}(2, \mathbb{R})$ transformations

$$
\tau \rightarrow \tau^{\prime}=\frac{a \tau+b}{c \tau+d}, \quad\left(\begin{array}{ll}
a & b \\
c & d
\end{array}\right) \in \operatorname{SL}(2, \mathbb{R}) .
$$

The functional $\int \mathrm{d}^{4} x e \mathfrak{L}$ proves to be Weyl invariant since the scalar field $\tau$ is inert under the Weyl transformations. The Weyl invariance follows from the fact that the FradkinTseytlin (FT) operator $[20,21]$

$$
\Delta_{0}=\left(\nabla^{a} \nabla_{a}\right)^{2}+2 \nabla^{a}\left(R_{a b} \nabla^{b}-\frac{1}{3} R \nabla_{a}\right)
$$

is conformal. ${ }^{3}$

Soon after Osborn's work [19] several attempts were made to extend his construction to supersymmetric case. So far no success has been achieved, mainly due to the following two

\footnotetext{
${ }^{2}$ Some of the relevant terms were missed in [16]. The complete action for $\mathcal{N}=4$ conformal supergravity was constructed in $[17,18]$.

${ }^{3}$ This operator was re-discovered by Paneitz in 1983 [22] and Riegert in 1984 [23].
} 
reasons. Firstly, the $\mathcal{N}=1$ supersymmetric extension of the FT operator was constructed only relatively recently $[24,25]$ (and its $\mathcal{N}=2$ cousin was presented in [25]). Secondly, the effective action corresponding to the vector multiplet model (4.1) proves to involve functional determinants of non-minimal differential operators, and these are much harder to evaluate in superspace than in ordinary quantum field theory. One of the goals of this work is to provide such a supersymmetric generalisation of [19]. Actually the scope of the present work is much broader, and in the remainder of this section we briefly describe the main results obtained below.

For every Kähler manifold $\mathcal{M}$, we construct a $\mathcal{N}=1$ locally superconformal fourderivative action which is formulated in terms of covariantly chiral superfields $\Phi^{I}$ taking values in $\mathcal{M}$. This action is invariant under (i) target-space isometries; and (ii) super-Weyl transformations of the supergravity multiplet. It is given by eq. (2.6) and involves two independent structures. The first term in the right-hand side of (2.6) contains a $\sigma$-model extension of the $\mathcal{N}=1$ supersymmetric FT operator. The second term in (2.6) involves the Riemann curvature tensor of $\mathcal{M} \cdot{ }^{4}$ In the case that $\mathcal{M}$ is the Hermitian symmetric space $\operatorname{Sp}(2 n, \mathbb{R}) / \mathrm{U}(n),(2.6)$ determines the logarithmically divergent parts of the effective action for $n$ vector multiplets coupled to $\Phi .^{5}$

We also present the general structure of $\mathcal{N}=1$ super-Weyl anomalies that contain weight-zero chiral multiplets $\Phi^{I}$ taking values in $\mathcal{M}$. The final expression for the anomaly is given by eq. (3.17). This anomaly is universal. Given a super-Weyl invariant theory coupled to background sources $\Phi^{I}$, the anomaly (3.17) should characterise the effective action obtained by integrating out the quantum fields. Analogous anomaly results for the $\mathcal{N}=0$ and $\mathcal{N}=2$ cases are known in the literature [28, 29], and this paper fills a gap concerning $\mathcal{N}=1$ superconformal symmetry.

When dealing with $\mathcal{N}=1$ local supersymmetry, me make use of the Grimm-WessZumino geometry [30,31] which underlies the Wess-Zumino formulation [32] for old minimal supergravity (see [33] for a review). Our two-component spinor notation and conventions follow [34]. The algebra of supergravity covariant derivatives is given in the appendix. To describe $\mathcal{N}=2$ (conformal) supergravity, the so-called SU(2) superspace formulation [35] is used.

\section{Superconformal higher-derivative actions}

In this section we describe $\mathcal{N}=1$ and $\mathcal{N}=2$ locally superconformal higher-derivative actions which are formulated in terms of chiral scalar superfields parametrising a Kähler manifold, for example the Hermitian symmetric spaces $\mathrm{Sp}(2 n, \mathbb{R}) / \mathrm{U}(n)$. Such actions occur, for instance, as the logarithmically divergent part of effective actions in $\operatorname{Sp}(2 n, \mathbb{R})$ dualityinvariant theories.

\footnotetext{
${ }^{4} \mathcal{N}=2$ supersymmetry uniquely fixes the relative coefficient $\alpha$ in (2.6) to be $\alpha=1$.

${ }^{5}$ Several global parametrisations of $\mathrm{Sp}(2 n, \mathbb{R}) / \mathrm{U}(n)$ and the corresponding Kähler potential are described, e.g., in [6, 26, 27].
} 


\section{$2.1 \quad$ Local $\mathcal{N}=1$ supersymmetry}

Let $\Phi$ be a covariantly chiral scalar superfield, $\overline{\mathcal{D}}^{\dot{\alpha}} \Phi=0$, which is neutral with respect to the super-Weyl transformations (A.4), $\delta_{\sigma} \Phi=0$. It was demonstrated in [25] that the following functional

$$
I=\frac{1}{16} \int \mathrm{d}^{4} x \mathrm{~d}^{2} \theta \mathrm{d}^{2} \bar{\theta} E\left\{\mathcal{D}^{2} \Phi \overline{\mathcal{D}}^{2} \bar{\Phi}-8 \mathcal{D}^{\alpha} \Phi G_{\alpha \dot{\alpha}} \overline{\mathcal{D}}^{\dot{\alpha}} \bar{\Phi}\right\}
$$

is super-Weyl invariant. The proof given in [25] makes use of the fact that $I$ transforms into

$$
\begin{aligned}
\delta_{\sigma} I & =\int \mathrm{d}^{4} x \mathrm{~d}^{2} \theta \mathrm{d}^{2} \bar{\theta} E\left\{\mathcal{D}^{\alpha} \sigma \mathcal{D}_{\alpha} \Phi \overline{\mathcal{D}}^{2} \bar{\Phi}+4 \mathrm{i} \mathcal{D}_{\alpha \dot{\alpha}} \sigma \mathcal{D}^{\alpha} \overline{\mathcal{D}}^{\dot{\alpha}} \bar{\Phi}+\text { c.c. }\right\} \\
& =-\frac{1}{8} \int \mathrm{d}^{4} x \mathrm{~d}^{2} \theta \mathrm{d}^{2} \bar{\theta} E \bar{\Phi}\left\{\overline{\mathcal{D}}^{2}\left(\mathcal{D}^{\alpha} \sigma \mathcal{D}_{\alpha} \Phi\right)+4 \mathrm{i} \overline{\mathcal{D}}^{\dot{\alpha}}\left(\mathcal{D}_{\alpha \dot{\alpha}} \sigma \mathcal{D}^{\alpha} \Phi\right)\right\}+\text { c.c. }=0 .
\end{aligned}
$$

The higher-derivative superconformal action $I$ possesses non-trivial generalisations which will be described below.

Now we assume, in addition, that $\Phi$ parametrises the upper half-plane, and therefore $(\Phi-\bar{\Phi})^{-1}$ exists. $^{6}$ Consider the following locally supersymmetric action

$$
\begin{aligned}
S= & -\frac{1}{16} \int \mathrm{d}^{4} x \mathrm{~d}^{2} \theta \mathrm{d}^{2} \bar{\theta} E \frac{1}{(\Phi-\bar{\Phi})^{2}}\left\{\nabla^{2} \Phi \bar{\nabla}^{2} \bar{\Phi}-8 \mathcal{D}^{\alpha} \Phi G_{\alpha \dot{\alpha}} \overline{\mathcal{D}}^{\dot{\alpha}} \bar{\Phi}\right\} \\
& +\frac{\alpha}{8} \int \mathrm{d}^{4} x \mathrm{~d}^{2} \theta \mathrm{d}^{2} \bar{\theta} E \frac{1}{(\Phi-\bar{\Phi})^{4}} \mathcal{D}^{\alpha} \Phi \mathcal{D}_{\alpha} \Phi \overline{\mathcal{D}}_{\dot{\alpha}} \bar{\Phi} \overline{\mathcal{D}}^{\dot{\alpha}} \bar{\Phi}
\end{aligned}
$$

where $\alpha$ is a real parameter, and

$$
\nabla^{2} \Phi=\mathcal{D}^{2} \Phi-2 \frac{\mathcal{D}^{\alpha} \Phi \mathcal{D}_{\alpha} \Phi}{\Phi-\bar{\Phi}}, \quad \bar{\nabla}^{2} \bar{\Phi}=\overline{\mathcal{D}}^{2} \bar{\Phi}+2 \frac{\overline{\mathcal{D}}_{\dot{\alpha}} \bar{\Phi} \overline{\mathcal{D}} \dot{\alpha} \bar{\Phi}}{\Phi-\bar{\Phi}}
$$

are $\mathrm{SL}(2, \mathbb{R})$ covariant derivatives. The action proves to be super-Weyl invariant. It is also invariant under fractional linear $\operatorname{SL}(2, \mathbb{R})$ transformations

$$
\Phi \rightarrow \Phi^{\prime}=\frac{a \Phi+b}{c \Phi+d}, \quad\left(\begin{array}{ll}
a & b \\
c & d
\end{array}\right) \in \operatorname{SL}(2, \mathbb{R}) .
$$

The above model can be generalised as follows. Let $K\left(\Phi^{I}, \bar{\Phi}^{\bar{J}}\right)$ be the Kähler potential of a Kähler manifold $\mathcal{M}$. We introduce a higher-derivative locally supersymmetric theory described in terms of covariantly chiral scalar superfields $\Phi^{I}, \overline{\mathcal{D}}^{\dot{\alpha}} \Phi^{I}=0$, which are neutral under the super-Weyl transformations, $\delta_{\sigma} \Phi^{I}=0$. The action is given by

$$
\begin{aligned}
S= & \frac{1}{16} \int \mathrm{d}^{4} x \mathrm{~d}^{2} \theta \mathrm{d}^{2} \bar{\theta} E g_{I \bar{J}}(\Phi, \bar{\Phi})\left\{\nabla^{2} \Phi^{I} \bar{\nabla}^{2} \bar{\Phi}^{\bar{J}}-8 G_{\alpha \dot{\alpha}} \mathcal{D}^{\alpha} \Phi^{I} \overline{\mathcal{D}}^{\dot{\alpha}} \bar{\Phi}^{\bar{J}}\right\} \\
& +\frac{\alpha}{16} \int \mathrm{d}^{4} x \mathrm{~d}^{2} \theta \mathrm{d}^{2} \bar{\theta} E R_{I \bar{J} K \bar{L}}(\Phi, \bar{\Phi}) \mathcal{D}^{\alpha} \Phi^{I} \mathcal{D}_{\alpha} \Phi^{K} \overline{\mathcal{D}}_{\dot{\alpha}} \bar{\Phi}^{\bar{J}} \overline{\mathcal{D}}^{\dot{\alpha}} \bar{\Phi}^{\bar{L}}
\end{aligned}
$$

\footnotetext{
${ }^{6}$ The axion $\mathfrak{a}(x)$ and dilaton $\varphi(x)$ are the component fields of $\Phi(x, \theta)$ defined by $\left.\Phi\right|_{\theta=0}=\mathfrak{a}+\mathrm{ie}^{-\varphi}$.
} 
where $g_{I \bar{J}}=\partial_{I} \partial_{\bar{J}} K$ is the Kähler metric, $R_{I \bar{J} K \bar{L}}(\Phi, \bar{\Phi})$ the Riemann curvature of the Kähler manifold, and

$$
\nabla^{2} \Phi^{I}=\overline{\mathcal{D}}^{2} \Phi^{I}+\Gamma_{K L}^{I} \mathcal{D}^{\alpha} \Phi^{K} \mathcal{D}_{\alpha} \Phi^{L}
$$

We recall that the Christoffel symbols $\Gamma_{K L}^{I}$ and the curvature $R_{I \bar{J} K \bar{L}}$ are given by the expressions

$$
\Gamma_{J K}^{I}=g^{I \bar{L}} \partial_{J} \partial_{K} \partial_{\bar{L}} K, \quad R_{I \bar{J} K \bar{L}}=\partial_{I} \partial_{K} \partial_{\bar{J}} \partial_{\bar{L}} K-g^{M \bar{N}} \partial_{I} \partial_{K} \partial_{\bar{N}} K \partial_{\bar{J}} \partial_{\bar{L}} \partial_{M} K
$$

It may be shown that the action (2.6) is super-Weyl invariant. This action is manifestly invariant under Kähler transformations

$$
K(\Phi, \bar{\Phi}) \rightarrow K(\Phi, \bar{\Phi})+\Lambda(\Phi)+\bar{\Lambda}(\bar{\Phi})
$$

with $\Lambda(\Phi)$ being an arbitrary holomorphic function.

\subsection{Local $\mathcal{N}=2$ supersymmetry}

Let $X$ be a covariantly chiral scalar superfield, $\overline{\mathcal{D}}_{i}^{\dot{\alpha}} X=0$, defined to be invariant under the $\mathcal{N}=2$ super-Weyl transformations [35], $\delta_{\Sigma} X=0$, with the super-Weyl parameter $\Sigma$ being covariantly chiral, $\overline{\mathcal{D}}_{i}^{\dot{\alpha}} \Sigma=0$. As in the $\mathcal{N}=1$ case, we assume that $(X-\bar{X})^{-1}$ exists. The following locally $\mathcal{N}=2$ supersymmetric action

$$
S=-\int \mathrm{d}^{4} x \mathrm{~d}^{4} \theta \mathrm{d}^{4} \bar{\theta} E \ln [\mathrm{i}(\bar{X}-X)]
$$

is obviously super-Weyl invariant. Moreover, it is invariant under $\operatorname{SL}(2, \mathbb{R})$ transformations

$$
X \rightarrow X^{\prime}=\frac{a X+b}{c X+d}, \quad\left(\begin{array}{ll}
a & b \\
c & d
\end{array}\right) \in \operatorname{SL}(2, \mathbb{R}) .
$$

The superfield Lagrangian in the higher-derivative action (2.10) is proportional to the Kähler potential $K(X, \bar{X})=-\ln \mathrm{i}(\bar{X}-X)$ of the Hermitian symmetric space $\mathrm{SL}(2, \mathbb{R}) / \mathrm{U}(1)$.

The above model has a natural generalisation given by

$$
S=\int \mathrm{d}^{4} x \mathrm{~d}^{4} \theta \mathrm{d}^{4} \bar{\theta} E K\left(X^{I}, \bar{X}^{\bar{J}}\right), \quad \overline{\mathcal{D}}_{i}^{\dot{\alpha}} X^{I}=0, \quad \delta_{\sigma} X^{I}=0,
$$

where the Kähler potential $K$ is the same as in (2.6). The action is super-Weyl invariant if the chiral multiplets $X^{I}$ are inert under the super-Weyl transformations. Using the standard properties of SU(2) superspace [35], it follows that the action (2.12) is superWeyl invariant. Due to the important property $[36,37]$

$$
\overline{\mathcal{D}}_{i}^{\dot{\alpha}} \Sigma=0 \quad \Longrightarrow \quad \int \mathrm{d}^{4} x \mathrm{~d}^{4} \theta \mathrm{d}^{4} \bar{\theta} E \Sigma=0,
$$

which holds for any covariantly chiral scalar $\Sigma$, we observe that the action (2.12) is also invariant under Kähler transformations

$$
K(X, \bar{X}) \rightarrow K(X, \bar{X})+\Lambda(X)+\bar{\Lambda}(\bar{X})
$$


with $\Lambda(X)$ being an arbitrary holomorphic function. It follows that (2.12) is invariant under isometry transformations of the Kähler space.

Superconformal actions of the form (2.12) were discussed in [38] in the rigid supersymmetric case, and later in the supergravity framework [25, 28, 39]. In ref. [38] $X$ was identified with the primary dimension-0 chiral scalar

$$
X=W^{-2} \bar{D}^{4} \ln \bar{W},
$$

where $W$ is the field strength of a vector multiplet, $\bar{D}_{i}^{\dot{\alpha}} W=0$, and

$$
\bar{D}^{4}=\frac{1}{16}\left(\bar{D}_{\hat{1}}\right)^{2}\left(\bar{D}_{\hat{2}}\right)^{2}=\frac{1}{48} \bar{D}^{i j} \bar{D}_{i j}, \quad \bar{D}^{i j}=\bar{D}_{\dot{\alpha}}^{i} \bar{D}^{j \dot{\alpha}}, \quad i, j=\underline{1}, \underline{2}
$$

is the chiral projection operator. The field strength $W$ is a reduced chiral superfield, $D^{i j} W=\bar{D}^{i j} \bar{W}$. For several Abelian field strengths $W^{I}$, higher-derivative actions

$$
S=\int \mathrm{d}^{4} x \mathrm{~d}^{4} \theta \mathrm{d}^{4} \bar{\theta} H\left(W^{I}, \bar{W}^{J}\right)
$$

were considered, e.g., in $[40,41]$. Locally supersymmetric extensions of such actions were analysed, e.g., in [39].

\subsection{Relating the $\mathcal{N}=2$ and $\mathcal{N}=1$ actions}

The models (2.6) and (2.12) are intimately related to each other. Their relationship is most transparent in Minkowski superspace, with $D_{\alpha}^{i}$ and $\bar{D}_{i}^{\dot{\alpha}}$ being the corresponding spinor covariant derivatives, $i=\underline{1}, \underline{2}$. Then the $\mathcal{N}=2$ chiral superfield $X^{I}, \bar{D}_{i}^{\dot{\alpha}} X^{I}=0$, is equivalent to three $\mathcal{N}=1$ chiral superfields $\Phi^{I}, \lambda_{\alpha}^{I}$ and $Z^{I}$ defined as follows:

$$
\Phi^{I}:=\left.X^{I}\right|_{\theta_{\underline{2}}=0}, \quad \sqrt{2} \Omega_{\alpha}^{I}:=\left.D_{\alpha}^{\underline{2}} X^{I}\right|_{\theta_{\underline{2}}=0}, \quad Z^{I}:=-\left.\frac{1}{4}\left(D^{2}\right)^{2} X^{I}\right|_{\theta_{2}=0} .
$$

The $\mathcal{N}=2$ supersymmetric action (2.12) reduces to $\mathcal{N}=1$ Minkowski superspace

$$
S=\int \mathrm{d}^{4} x \mathrm{~d}^{4} \theta \mathrm{d}^{4} \bar{\theta} K(X, \bar{X})=\left.\frac{1}{16} \int \mathrm{d}^{4} x \mathrm{~d}^{2} \theta \mathrm{d}^{2} \bar{\theta}\left(D^{2}\right)^{2}\left(\bar{D}_{\underline{2}}\right)^{2} K(X, \bar{X})\right|_{\theta_{\underline{2}}=\bar{\theta} \underline{\underline{\theta}}=0} .
$$

Now direct calculations give

$$
\begin{aligned}
S= & \frac{1}{16} \int \mathrm{d}^{4} x \mathrm{~d}^{2} \theta \mathrm{d}^{2} \bar{\theta}\left\{g_{I \bar{J}} \nabla^{2} \Phi^{I} \bar{\nabla}^{2} \bar{\Phi}^{\bar{J}}+R_{I \bar{J} K \bar{L}} D^{\alpha} \Phi^{I} D_{\alpha} \Phi^{K} \bar{D}_{\dot{\alpha}} \bar{\Phi}^{\bar{I}} \bar{D}^{\dot{\alpha}} \bar{\Phi}^{\bar{L}}\right\} \\
& +\int \mathrm{d}^{4} x \mathrm{~d}^{2} \theta \mathrm{d}^{2} \bar{\theta} g_{I \bar{J}}\left\{\mathbb{Z}^{I} \overline{\mathbb{Z}}^{\bar{J}}-\mathrm{i} \Omega^{I \alpha} \nabla_{\alpha \dot{\alpha}} \bar{\Omega}^{\bar{J} \dot{\alpha}}\right\},
\end{aligned}
$$

where we have defined

$$
\mathbb{Z}^{I}=Z^{I}-\frac{1}{4} \Gamma_{J K}^{I} \Omega^{I \alpha} \Omega_{\alpha}^{K}, \quad \nabla_{\alpha \dot{\alpha}} \bar{\Omega}^{\bar{I} \dot{\alpha}}=\partial_{\alpha \dot{\alpha}} \bar{\Omega}^{\bar{J} \dot{\alpha}}+\Gamma_{\bar{J} \bar{K}}^{\bar{I}} \partial_{\alpha \dot{\alpha}} \bar{\Phi}^{\bar{J}} \bar{\Omega}^{\bar{K} \dot{\alpha}} .
$$

The superfield $\mathbb{Z}^{I}$ transforms as a target-space vector under holomorphic reparametrisations of the Kähler manifold, but it is not chiral unlike $Z^{I}$. The first term in (2.20) is the flatsuperspace version of (2.6) with $a=b$. Thus $\mathcal{N}=2$ supersymmetry fixes the relative coefficient between the two structures in (2.6). 
The curved superspace version of the quadratic spinor sector in (2.20) has recently been described in [42]. That work introduced the model for a covariantly chiral spinor $\Omega_{\alpha}$ that is primary of dimension $1 / 2$,

$$
\overline{\mathcal{D}}^{\dot{\beta}} \Omega_{\alpha}=0, \quad \delta_{\sigma} \Omega_{\alpha}=\frac{1}{2} \sigma \Omega_{\alpha} .
$$

The corresponding action

$$
S[\Omega, \bar{\Omega}]=-\int \mathrm{d}^{4} x \mathrm{~d}^{2} \theta \mathrm{d}^{2} \bar{\theta} E \Omega^{\alpha}\left(\mathrm{i} \mathcal{D}_{\alpha \dot{\alpha}}-G_{\alpha \dot{\alpha}}\right) \bar{\Omega}^{\dot{\alpha}}
$$

is super-Weyl invariant, as follows from (A.4) and (A.5b). This model is a special representative of the family of superconformal higher-derivative actions [42] formulated in terms of covariantly chiral rank- $n$ spinors $\Omega_{\alpha(n)}:=\Omega_{\left(\alpha_{1} \ldots \alpha_{n}\right)}$ of dimension $(1-n / 2)$,

$$
\overline{\mathcal{D}}^{\dot{\beta}} \Omega_{\alpha(n)}=0, \quad \delta_{\sigma} \Omega_{\alpha(n)}=\frac{1}{2}(2-n) \sigma \Omega_{\alpha(n)} .
$$

\section{Super-Weyl anomalies}

This section is devoted to $\mathcal{N}=1$ and $\mathcal{N}=2$ super-Weyl anomalies that contain weight-zero superconformal chiral multiplets parametrising a Kähler manifold $\mathcal{M}$.

\section{$3.1 \mathcal{N}=1$ super-Weyl anomalies}

There are two different contributions to $\mathcal{N}=1$ super-Weyl anomalies. One of them is given in terms of the supergravity multiplet. Modulo cohomologically trivial contributions, the super-Weyl variation of the effective action is

$$
\begin{aligned}
\delta_{\sigma} \Gamma= & 2(a-c) \int \mathrm{d}^{4} x \mathrm{~d}^{2} \theta \mathcal{E} \sigma W^{\alpha \beta \gamma} W_{\alpha \beta \gamma}+\text { c.c. } \\
& +2 a \int \mathrm{d}^{4} x \mathrm{~d}^{2} \theta \mathrm{d}^{2} \bar{\theta} E(\sigma+\bar{\sigma})\left(G^{a} G_{a}+2 R \bar{R}\right) .
\end{aligned}
$$

The functional structures which contribute to the anomaly (3.1) were contained in the chiral super- $b_{4}$ coefficient computed in 1983 by McArthur [43, 44]. His work was followed by the cohomological analysis of Bonora, Pasti and Tonin [45] who arrived at the same structures. The explicit calculations of the super-Weyl anomalies for the scalar and vector multiplets were carried out in [46]. Various effective actions generating the anomaly (3.1) were proposed in [24, 47-49].

The second type of super-Weyl anomalies is determined by local couplings in a superconformal field theory. In general, such an anomaly is given by

$$
\begin{aligned}
\delta_{\sigma} \widetilde{\Gamma}= & \frac{1}{16} \int \mathrm{d}^{4} x \mathrm{~d}^{2} \theta \mathrm{d}^{2} \bar{\theta} E \sigma\left\{K_{\bar{I}}(\Phi, \bar{\Phi})\left(\mathcal{D}^{2} \overline{\mathcal{D}}^{2} \bar{\Phi}^{\bar{I}}+8 \mathcal{D}^{\alpha}\left(G_{\alpha \dot{\alpha}} \overline{\mathcal{D}}^{\dot{\alpha}} \bar{\Phi}^{\bar{I}}\right)\right)\right. \\
& \left.-8 K_{\bar{I} \bar{J}}(\Phi, \bar{\Phi})\left(\mathcal{D}^{\alpha \dot{\alpha}} \bar{\Phi}^{\bar{I}} \mathcal{D}_{\alpha \dot{\alpha}} \bar{\Phi}^{\bar{J}}-\bar{R} \overline{\mathcal{D}}_{\dot{\alpha}} \bar{\Phi}^{\bar{I}} \mathcal{D}^{\dot{\alpha}} \bar{\Phi}^{\bar{J}}\right)\right\}+ \text { c.c. } \\
& +\frac{\alpha}{16} \int \mathrm{d}^{4} x \mathrm{~d}^{2} \theta \mathrm{d}^{2} \bar{\theta} E(\sigma+\bar{\sigma}) R_{I \bar{J} K \bar{L}}(\Phi, \bar{\Phi}) \mathcal{D}^{\alpha} \Phi^{I} \mathcal{D}_{\alpha} \Phi^{K} \overline{\mathcal{D}}_{\dot{\alpha}} \bar{\Phi}^{\bar{J}} \overline{\mathcal{D}}^{\dot{\alpha}} \bar{\Phi}^{\bar{L}}
\end{aligned}
$$


compare with the $\mathcal{N}=2$ super-Weyl anomaly (3.20). This anomaly obeys the Wess-Zumino consistency condition, $\left[\delta_{\sigma_{2}}, \delta_{\sigma_{1}}\right] \widetilde{\Gamma}=0$, since

$$
\delta_{\sigma_{2}} \delta_{\sigma_{1}} \widetilde{\Gamma}=0,
$$

as a consequence of the following identity [24]:

$$
\begin{aligned}
\delta_{\sigma}\left\{\mathcal{D}^{2} \overline{\mathcal{D}}^{2} \bar{\Phi}+8 \mathcal{D}^{\alpha}\left(G_{\alpha \dot{\alpha}} \overline{\mathcal{D}}^{\dot{\alpha}} \bar{\Phi}\right)\right\}= & (\sigma+\bar{\sigma})\left\{\mathcal{D}^{2} \overline{\mathcal{D}}^{2} \bar{\Phi}+8 \mathcal{D}^{\alpha}\left(G_{\alpha \dot{\alpha}} \overline{\mathcal{D}}^{\dot{\alpha}} \bar{\Phi}\right)\right\} \\
& 2 \overline{\mathcal{D}}_{\dot{\alpha}}\left\{\left(\overline{\mathcal{D}}^{\dot{\alpha}} \bar{\Phi}\right) \mathcal{D}^{2} \sigma+4 \mathrm{i}\left(\mathcal{D}^{\alpha \dot{\alpha}} \bar{\Phi}\right) \mathcal{D}_{\alpha} \sigma\right\}
\end{aligned}
$$

At this point an important comment should be made. It follows from (3.4) that the operator

$$
\Delta_{\mathrm{c}} \bar{\Phi}:=-\frac{1}{64}\left(\overline{\mathcal{D}}^{2}-4 R\right)\left\{\mathcal{D}^{2} \overline{\mathcal{D}}^{2} \bar{\Phi}+8 \mathcal{D}^{\alpha}\left(G_{\alpha \dot{\alpha}} \overline{\mathcal{D}}^{\dot{\alpha}} \bar{\Phi}\right)\right\}, \quad \overline{\mathcal{D}}^{\dot{\alpha}} \Delta_{\mathrm{c}} \bar{\Phi}=0
$$

is superconformal in the sense that it has the super-Weyl transformation law

$$
\delta_{\sigma} \Delta_{\mathrm{c}} \bar{\Phi}=3 \sigma \Delta_{\mathrm{c}} \bar{\Phi} .
$$

$\Delta_{\mathrm{c}}$ is the $\mathcal{N}=1$ supersymmetric extension of the Fradkin-Tseytlin operator (1.5). This operator played important roles in the analyses carried out in $[24,50]$.

The super-Weyl anomaly (3.2) may be rewritten in a different form,

$$
\begin{aligned}
\delta_{\sigma} \widetilde{\Gamma}= & \frac{1}{16} \int \mathrm{d}^{4} x \mathrm{~d}^{2} \theta \mathrm{d}^{2} \bar{\theta} E\left\{\sigma K_{\bar{I}}(\Phi, \bar{\Phi})\left(\mathcal{D}^{2} \overline{\mathcal{D}}^{2} \bar{\Phi}^{\bar{I}}+8 \mathcal{D}^{\alpha}\left(G_{\alpha \dot{\alpha}} \overline{\mathcal{D}}^{\dot{\alpha}} \bar{\Phi}^{\bar{I}}\right)\right)\right. \\
& \left.+\mathcal{D}^{2}\left(\sigma K_{\bar{I} \bar{J}}(\Phi, \bar{\Phi})\right) \overline{\mathcal{D}}_{\dot{\alpha}} \bar{\Phi}^{\bar{I}} \overline{\mathcal{D}}^{\dot{\alpha}} \bar{\Phi}^{\bar{J}}\right\}+ \text { c.c. } \\
& +\frac{\alpha}{16} \int \mathrm{d}^{4} x \mathrm{~d}^{2} \theta \mathrm{d}^{2} \bar{\theta} E(\sigma+\bar{\sigma}) R_{I \bar{J} K \bar{L}}(\Phi, \bar{\Phi}) \mathcal{D}^{\alpha} \Phi^{I} \mathcal{D}_{\alpha} \Phi^{K} \overline{\mathcal{D}}_{\dot{\alpha}} \bar{\Phi}^{\bar{I}} \overline{\mathcal{D}}^{\dot{\alpha}} \bar{\Phi}^{\bar{L}}
\end{aligned}
$$

which is more useful for analysing the behaviour of $\delta_{\sigma} \widetilde{\Gamma}$ under Kähler transformations (2.9). One obtains

$$
\begin{aligned}
\delta_{\Lambda} \delta_{\sigma} \widetilde{\Gamma}= & \frac{1}{16} \int \mathrm{d}^{4} x \mathrm{~d}^{2} \theta \mathrm{d}^{2} \bar{\theta} E\left\{\sigma \bar{\Lambda}_{\bar{I}}(\bar{\Phi})\left(\mathcal{D}^{2} \overline{\mathcal{D}}^{2} \bar{\Phi}^{\bar{I}}+8 \mathcal{D}^{\alpha}\left(G_{\alpha \dot{\alpha}} \overline{\mathcal{D}}^{\dot{\alpha}} \bar{\Phi}^{\bar{I}}\right)\right)\right. \\
& \left.+\left(\mathcal{D}^{2} \sigma\right) \bar{\Lambda}_{\bar{I} \bar{J}}(\bar{\Phi}) \overline{\mathcal{D}}_{\dot{\alpha}} \bar{\Phi}^{\bar{I}} \overline{\mathcal{D}}^{\dot{\alpha}} \bar{\Phi}^{\bar{J}}\right\}+ \text { c.c. } \\
= & \frac{1}{16} \int \mathrm{d}^{4} x \mathrm{~d}^{2} \theta \mathrm{d}^{2} \bar{\theta} E \bar{\Lambda}(\bar{\Phi})\left(\overline{\mathcal{D}}^{2} \mathcal{D}^{2} \sigma-8 \overline{\mathcal{D}}^{\dot{\alpha}}\left(G_{\alpha \dot{\alpha}} \mathcal{D}^{\alpha} \sigma\right)\right)+\text { c.c. }
\end{aligned}
$$

Making use of the identity [24]

$$
\begin{aligned}
\delta_{\sigma}\left\{G^{a} G_{a}\right. & \left.+2 R \bar{R}-\frac{1}{4} \overline{\mathcal{D}}^{2} \bar{R}\right\}=(\sigma+\bar{\sigma})\left\{G^{a} G_{a}+2 R \bar{R}-\frac{1}{4} \overline{\mathcal{D}}^{2} \bar{R}\right\} \\
& -\frac{1}{16}\left(\overline{\mathcal{D}}^{2} \mathcal{D}^{2} \sigma-8 \overline{\mathcal{D}}^{\dot{\alpha}}\left(G_{\alpha \dot{\alpha}} \mathcal{D}^{\alpha} \sigma\right)\right)+\frac{1}{2} \mathcal{D}^{\alpha}\left(R \mathcal{D}_{\alpha} \sigma-G_{\alpha \dot{\alpha}} \overline{\mathcal{D}}^{\dot{\alpha}} \bar{\sigma}\right),
\end{aligned}
$$


it follows that (3.8) can be recast as the super-Weyl variation of a local functional,

$$
\begin{aligned}
\delta_{\Lambda} \delta_{\sigma} \widetilde{\Gamma} & =-\delta_{\sigma} \int \mathrm{d}^{4} x \mathrm{~d}^{2} \theta \mathrm{d}^{2} \bar{\theta} E \Lambda(\Phi)\left(G^{a} G_{a}+2 R \bar{R}-\frac{1}{4} \mathcal{D}^{2} R\right)+\text { c.c. } \\
& =-\delta_{\sigma} \delta_{\Lambda} \int \mathrm{d}^{4} x \mathrm{~d}^{2} \theta \mathrm{d}^{2} \bar{\theta} E K(\Phi, \bar{\Phi})\left\{G^{a} G_{a}+2 R \bar{R}-\frac{1}{4}\left(\mathcal{D}^{2} R+\overline{\mathcal{D}}^{2} \bar{R}\right)\right\}
\end{aligned}
$$

and therefore

$$
\delta_{\Lambda} \delta_{\sigma}\left\{\widetilde{\Gamma}+\int \mathrm{d}^{4} x \mathrm{~d}^{2} \theta \mathrm{d}^{2} \bar{\theta} E K(\Phi, \bar{\Phi})\left(G^{a} G_{a}+2 R \bar{R}-\frac{1}{4}\left(\mathcal{D}^{2} R+\overline{\mathcal{D}}^{2} \bar{R}\right)\right)\right\}=0 .
$$

If we now introduce the functional

$$
\widetilde{\widetilde{\Gamma}}:=\widetilde{\Gamma}+\int \mathrm{d}^{4} x \mathrm{~d}^{2} \theta \mathrm{d}^{2} \bar{\theta} E K(\Phi, \bar{\Phi})\left(G^{a} G_{a}+2 R \bar{R}-\frac{1}{4}\left(\mathcal{D}^{2} R+\overline{\mathcal{D}}^{2} \bar{R}\right)\right),
$$

then its super-Weyl variation may be shown to be

$$
\begin{aligned}
\delta_{\sigma} \widetilde{\widetilde{\Gamma}}= & -\int \mathrm{d}^{4} x \mathrm{~d}^{2} \theta \mathrm{d}^{2} \bar{\theta} E \sigma g_{I \bar{J}}(\Phi, \bar{\Phi})\left\{\frac{1}{16} \nabla^{2} \Phi^{I} \bar{\nabla}^{2} \bar{\Phi}^{\bar{J}}+G_{\alpha \dot{\alpha}} \mathcal{D}^{\alpha} \Phi^{I} \overline{\mathcal{D}}^{\dot{\alpha}} \bar{\Phi}^{\bar{J}}\right. \\
& \left.-\frac{\mathrm{i}}{2} \mathcal{D}^{\alpha} \Phi^{I} \nabla_{\alpha \dot{\alpha}} \overline{\mathcal{D}}^{\dot{\alpha}} \bar{\Phi}^{\bar{J}}\right\}+ \text { c.c. } \\
& +\frac{\alpha-1}{16} \int \mathrm{d}^{4} x \mathrm{~d}^{2} \theta \mathrm{d}^{2} \bar{\theta} E(\sigma+\bar{\sigma}) R_{I \bar{J} K \bar{L}}(\Phi, \bar{\Phi}) \mathcal{D}^{\alpha} \Phi^{I} \mathcal{D}_{\alpha} \Phi^{K} \overline{\mathcal{D}}_{\dot{\alpha}} \bar{\Phi}^{\bar{J}} \overline{\mathcal{D}}^{\dot{\alpha}} \bar{\Phi}^{\bar{L}},
\end{aligned}
$$

where $\nabla^{2} \Phi^{I}$ is given by $(2.7)$, and $\nabla_{\alpha \dot{\alpha}} \overline{\mathcal{D}}^{\dot{\alpha}} \bar{\Phi}^{\bar{I}}$ is defined as

$$
\nabla_{\alpha \dot{\alpha}} \overline{\mathcal{D}}^{\dot{\alpha}} \bar{\Phi}^{\bar{I}}=\mathcal{D}_{\alpha \dot{\alpha}} \overline{\mathcal{D}}^{\dot{\alpha}} \bar{\Phi}^{\bar{I}}+\Gamma_{\bar{J} \bar{K}}^{\bar{I}} \mathcal{D}_{\alpha \dot{\alpha}} \bar{\Phi}^{\bar{J}} \overline{\mathcal{D}}^{\dot{\alpha}} \bar{\Phi}^{\bar{K}}
$$

The super-Weyl anomaly (3.13) is manifestly Kähler invariant.

Finally, we can recast the anomaly (3.13) in a more compact form by making use of the identity

$$
\begin{aligned}
\delta_{\sigma} \int \mathrm{d}^{4} x \mathrm{~d}^{2} \theta \mathrm{d}^{2} \bar{\theta} E g_{I \bar{J}} G_{\alpha \dot{\alpha}} \mathcal{D}^{\alpha} \Phi^{I} \overline{\mathcal{D}}^{\dot{\alpha}} \bar{\Phi}^{\bar{J}} & =-\mathrm{i} \int \mathrm{d}^{4} x \mathrm{~d}^{2} \theta \mathrm{d}^{2} \bar{\theta} E \sigma g_{I \bar{J}} \mathcal{D}^{\alpha} \Phi^{I} \nabla_{\alpha \dot{\alpha}} \overline{\mathcal{D}}^{\dot{\alpha}} \bar{\Phi}^{\bar{J}} \\
+\frac{1}{4} \int \mathrm{d}^{4} x \mathrm{~d}^{2} \theta \mathrm{d}^{2} \bar{\theta} E \sigma\left\{g_{I \bar{J}} \nabla^{2} \Phi^{I} \bar{\nabla}^{2} \bar{\Phi}^{\bar{J}}\right. & +R_{I \bar{J} K \bar{L}} \mathcal{D}^{\alpha} \Phi^{I} \mathcal{D}_{\alpha} \Phi^{K} \overline{\mathcal{D}}_{\dot{\alpha}} \bar{\Phi}^{\bar{J}} \overline{\mathcal{D}}^{\dot{\alpha}} \bar{\Phi}^{\bar{L}} \\
& \left.+4 g_{I \bar{J}} G_{\alpha \dot{\alpha}} \mathcal{D}^{\alpha} \Phi^{I} \overline{\mathcal{D}}^{\dot{\alpha}} \bar{\Phi}^{\bar{J}}\right\}+ \text { c.c. }
\end{aligned}
$$

This identity shows that the functional

$$
\Gamma:=\widetilde{\widetilde{\Gamma}}+\frac{1}{2} \int \mathrm{d}^{4} x \mathrm{~d}^{2} \theta \mathrm{d}^{2} \bar{\theta} E g_{I \bar{J}}(\Phi, \bar{\Phi}) G_{\alpha \dot{\alpha}} \mathcal{D}^{\alpha} \Phi^{I} \overline{\mathcal{D}}^{\dot{\alpha}} \bar{\Phi}^{\bar{J}}
$$

has the following super-Weyl variation

$$
\begin{aligned}
\delta_{\sigma} \Gamma= & \frac{1}{16} \int \mathrm{d}^{4} x \mathrm{~d}^{2} \theta \mathrm{d}^{2} \bar{\theta} E \sigma g_{I \bar{J}}(\Phi, \bar{\Phi})\left\{\nabla^{2} \Phi^{I} \bar{\nabla}^{2} \bar{\Phi}^{\bar{J}}-8 G_{\alpha \dot{\alpha}} \mathcal{D}^{\alpha} \Phi^{I} \overline{\mathcal{D}}^{\dot{\alpha}} \bar{\Phi}^{\bar{J}}\right\}+\text { c.c. } \\
& +\frac{\alpha+1}{16} \int \mathrm{d}^{4} x \mathrm{~d}^{2} \theta \mathrm{d}^{2} \bar{\theta} E(\sigma+\bar{\sigma}) R_{I \bar{J} K \bar{L}}(\Phi, \bar{\Phi}) \mathcal{D}^{\alpha} \Phi^{I} \mathcal{D}_{\alpha} \Phi^{K} \overline{\mathcal{D}}_{\dot{\alpha}} \bar{\Phi}^{\bar{J}} \overline{\mathcal{D}}^{\dot{\alpha}} \bar{\Phi}^{\bar{L}} .
\end{aligned}
$$

This is our final expression for the super-Weyl anomaly determined by local couplings in $\mathcal{N}=1$ superconformal field theories. 


\section{$3.2 \mathcal{N}=2$ super-Weyl anomalies}

It is a curious fact that, in the literature, the general structure of $\mathcal{N}=2$ super-Weyl anomalies has been understood better than in the $\mathcal{N}=1$ case. Unlike the $\mathcal{N}=1$ case, however, no supergraph calculation of the $\mathcal{N}=2$ super-Weyl anomalies has yet appeared.

There are two different contributions to the $\mathcal{N}=2$ super-Weyl anomaly. One of them is given in terms of the supergravity multiplet [51] and has the form

$$
\delta_{\Sigma} \Gamma=(c-a) \int \mathrm{d}^{4} x \mathrm{~d}^{4} \theta \mathcal{E} \Sigma W^{\alpha \beta} W_{\alpha \beta}+a \int \mathrm{d}^{4} x \mathrm{~d}^{4} \theta \mathcal{E} \Sigma \Xi+\text { c.c. },
$$

for some anomaly coefficients $a$ and $c$. Within the SU(2) superspace approach [35], the super-Weyl parameter $\Sigma$ is an unrestricted chiral superfield. The chiral rank-2 spinor $W_{\alpha \beta}=W_{\beta \alpha}$ in (3.18) is the $\mathcal{N}=2$ super-Weyl tensor. The composite chiral scalar $\Xi$ in the second term of (3.18) is the $\mathcal{N}=2$ counterpart of the local operator [24]

$$
\hat{Q}=-\frac{1}{4}\left(\overline{\mathcal{D}}^{2}-4 R\right)\left\{G^{a} G_{a}+2 R \bar{R}-\frac{1}{4} \mathcal{D}^{2} R\right\}
$$

in $\mathcal{N}=1$ supergravity. ${ }^{7}$ The composite $\Xi$ has several fundamental properties $[25,51]$ which are also important for the construction of $\mathcal{N}=2$ Liouville SCFT in four dimensions [53].

The other sector of the $\mathcal{N}=2$ super-Weyl anomaly is determined by local couplings in a superconformal field theory. According to $[28,29]$, it is given by

$$
\delta_{\Sigma} \Gamma=\int \mathrm{d}^{4} x \mathrm{~d}^{4} \theta \mathrm{d}^{4} \bar{\theta} E(\Sigma+\bar{\Sigma}) K(X, \bar{X})
$$

where the Kähler potential $K(X, \bar{X})$ is the same as in (2.12). Since the chiral scalars $X^{I}$ are inert under the super-Weyl transformations, the anomaly clearly satisfies the WessZumino consistency condition. The right-hand side of (3.20) is not invariant under Kähler transformations, unlike the $\mathcal{N}=1$ super-Weyl anomaly (3.17). However, the $\mathcal{N}=2$ super-Weyl anomaly is invariant under a joint Kähler-Weyl transformation. A detailed analysis of the anomaly (3.20) is given in the original publications [28, 29] to which the reader is referred for the technical details. In the case of the Hermitian symmetric spaces $\mathrm{Sp}(2 n, \mathbb{R}) / \mathrm{U}(n)$, the Kähler potential $K(X, \bar{X})$ can be chosen to be a globally defined function on the target space (see, e.g., [27]). Choosing a different globally defined Kähler potential corresponds to a different scheme.

\section{Quantisation}

We consider the model for a massless vector multiplet coupled to a dilaton-axion chiral superfield $\Phi, \overline{\mathcal{D}}^{\dot{\alpha}} \Phi=0$, in curved superspace. Its dynamics is described by the action

$$
S[V ; \Phi, \bar{\Phi}]=-\frac{\mathrm{i}}{4} \int \mathrm{d}^{4} x \mathrm{~d}^{2} \theta \mathcal{E} \Phi W^{\alpha}(V) W_{\alpha}(V)+\text { c.c. },
$$

\footnotetext{
${ }^{7} \hat{Q}$ is known in the literature as the $\mathcal{N}=1$ supersymmetric $Q$-curvature [50,52].
} 
where $W_{\alpha}(V)=-\frac{1}{4}\left(\overline{\mathcal{D}}^{2}-4 R\right) \mathcal{D}_{\alpha} V$. The chiral field strength $W_{\alpha}(V)$ and the action are invariant under gauge transformations

$$
\delta_{\lambda} V=\lambda+\bar{\lambda}, \quad \overline{\mathcal{D}}^{\dot{\alpha}} \lambda=0 .
$$

The gauge prepotential $V$ is chosen to be super-Weyl inert, $\delta_{\sigma} V=0$, which implies

$$
\delta_{\sigma} W_{\alpha}(V)=\frac{3}{2} \sigma W_{\alpha}(V) .
$$

As a consequence, the action (4.1) is super-Weyl invariant. In addition, the model possesses $\mathrm{SL}(2, \mathbb{R})$ duality. ${ }^{8}$ The duality group acts on $\Phi$ by fractional linear transformations (2.5).

Making use of the chiral action rule

$$
\int \mathrm{d}^{4} x \mathrm{~d}^{2} \theta \mathrm{d}^{2} \bar{\theta} E \mathcal{L}=-\frac{1}{4} \int \mathrm{d}^{4} x \mathrm{~d}^{2} \theta \mathcal{E}\left(\overline{\mathcal{D}}^{2}-4 R\right) \mathcal{L}
$$

the action (4.1) can be rewritten in the form

$$
\begin{aligned}
S= & \frac{1}{16} \int \mathrm{d}^{4} x \mathrm{~d}^{2} \theta \mathrm{d}^{2} \bar{\theta} E V\left\{\Xi \mathcal{D}^{\alpha}\left(\overline{\mathcal{D}}^{2}-4 R\right) \mathcal{D}_{\alpha}\right. \\
& \left.+\left(\mathcal{D}^{\alpha} \Xi\right)\left(\overline{\mathcal{D}}^{2}-4 R\right) \mathcal{D}_{\alpha}+\left(\overline{\mathcal{D}}_{\dot{\alpha}} \Xi\right)\left(\mathcal{D}^{2}-4 \bar{R}\right) \overline{\mathcal{D}}^{\dot{\alpha}}\right\} V, \quad \Xi \equiv \mathrm{i}(\bar{\Phi}-\Phi) .
\end{aligned}
$$

We choose the following gauge-fixing term

$$
S_{\text {G.F. }}=-\frac{1}{16} \int \mathrm{d}^{4} x \mathrm{~d}^{2} \theta \mathrm{d}^{2} \bar{\theta} E \Xi\left[\left(\overline{\mathcal{D}}^{2}-4 R\right) V\right]\left[\left(\mathcal{D}^{2}-4 \bar{R}\right) V\right] .
$$

Then the complete action is given by

$$
S+S_{\text {G.F. }}=-\frac{1}{2} \int \mathrm{d}^{4} x \mathrm{~d}^{2} \theta \mathrm{d}^{2} \bar{\theta} E V \boldsymbol{\Delta}_{\mathrm{v}} V,
$$

where we have introduced the second-order operator

$$
\begin{aligned}
\boldsymbol{\Delta}_{\mathrm{v}}=\Xi \square_{\mathrm{v}} & -\frac{\mathrm{i}}{4}\left(\overline{\mathcal{D}}^{\dot{\alpha}} \Xi\right)\left\{\mathcal{D}_{\alpha \dot{\alpha}}, \mathcal{D}^{\alpha}\right\}-\frac{\mathrm{i}}{4}\left(\mathcal{D}^{\alpha} \Xi\right)\left\{\mathcal{D}_{\alpha \dot{\alpha}}, \overline{\mathcal{D}}^{\dot{\alpha}}\right\} \\
& +\frac{1}{16}\left(\overline{\mathcal{D}}^{2} \Xi\right)\left(\mathcal{D}^{2}-4 \bar{R}\right)+\frac{1}{16}\left(\mathcal{D}^{2} \Xi\right)\left(\overline{\mathcal{D}}^{2}-4 R\right) \\
& -\frac{1}{2}\left(\mathcal{D}^{\alpha} \Xi\right)\left(\mathcal{D}_{\alpha} R\right)-\frac{1}{2}\left(\overline{\mathcal{D}}_{\dot{\alpha}} \Xi\right)\left(\overline{\mathcal{D}}^{\dot{\alpha}} \bar{R}\right),
\end{aligned}
$$

in which the operator $\square_{\mathrm{v}}$ in the first term is

$$
\begin{aligned}
\square_{\mathrm{v}}= & -\frac{1}{8} \mathcal{D}^{\alpha}\left(\overline{\mathcal{D}}^{2}-4 R\right) \mathcal{D}_{\alpha}+\frac{1}{16}\left\{\overline{\mathcal{D}}^{2}-4 R, \mathcal{D}^{2}-4 \bar{R}\right\} \\
= & \mathcal{D}^{a} \mathcal{D}_{a}-\frac{1}{4} G^{\alpha \dot{\alpha}}\left[\mathcal{D}_{\alpha}, \overline{\mathcal{D}}_{\dot{\alpha}}\right]-\frac{1}{4}\left(\mathcal{D}^{\alpha} R\right) \mathcal{D}_{\alpha}-\frac{1}{4}\left(\overline{\mathcal{D}}_{\dot{\alpha}} \bar{R}\right) \overline{\mathcal{D}}^{\dot{\alpha}} \\
& -\frac{1}{4}\left(\mathcal{D}^{2} R\right)-\frac{1}{4}\left(\overline{\mathcal{D}}^{2} \bar{R}\right)+2 R \bar{R} .
\end{aligned}
$$

The operator $\boldsymbol{\Delta}_{\mathrm{v}}$ reduces to $\square_{\mathrm{v}}$ in the $\Xi=1$ case.

\footnotetext{
${ }^{8}$ Within the $\mathcal{N}=1$ Poincaré supersymmetry, $\operatorname{SL}(2, \mathbb{R})$ duality invariant couplings of the dilaton-axion multiplet to general models for self-dual supersymmetric nonlinear electrodynamics were described in [12], while the case of the supersymmetric Born-Infeld action [54, 55] was first considered in [56]. The results of [12] were generalised to supergravity in [57].
} 
A useful gauge condition is

$$
\kappa(V)=-\frac{1}{4}\left(\overline{\mathcal{D}}^{2}-4 R\right) V+\eta, \quad \overline{\mathcal{D}}^{\dot{\alpha}} \eta=0
$$

with $\eta$ a background chiral superfield. Then the Faddeev-Popov operator is $H^{(R)}$, where, in general, $H^{(\psi)}$ denotes the following operator

$$
H^{(\psi)}=\left(\begin{array}{cc}
\psi & -\frac{1}{4}\left(\overline{\mathcal{D}}^{2}-4 R\right) \\
-\frac{1}{4}\left(\mathcal{D}^{2}-4 \bar{R}\right) & \bar{\psi}
\end{array}\right), \quad \overline{\mathcal{D}}^{\dot{\alpha}} \psi=0 .
$$

For the effective action we obtain

$$
\mathrm{e}^{\mathrm{i} \Gamma_{\mathrm{v}}[\Phi, \bar{\Phi}]}=\int \mathrm{D} V \delta_{+}\left[\eta-\frac{1}{4}\left(\overline{\mathcal{D}}^{2}-4 R\right) V\right] \delta_{-}\left[\bar{\eta}-\frac{1}{4}\left(\mathcal{D}^{2}-4 \bar{R}\right) V\right] \operatorname{Det} H^{(R)} \mathrm{e}^{\mathrm{i} S[V ; \Phi, \bar{\Phi}]},
$$

where Det $H^{(R)}$ stands for the ghost determinant. Since the right-hand side is independent of $\eta$ and $\bar{\eta}$, we can average it over these superfields with weight

$$
\operatorname{Det}^{1 / 2} \boldsymbol{H}_{\Xi} \mathrm{e}^{-\mathrm{i} S[\eta, \bar{\eta} ; \Xi]}, \quad S[\eta, \bar{\eta} ; \Xi]=\int \mathrm{d}^{4} x \mathrm{~d}^{2} \theta \mathrm{d}^{2} \bar{\theta} E \Xi \bar{\eta} \eta,
$$

where $\boldsymbol{H}_{\Xi}$ denotes the following operator

$$
\boldsymbol{H}_{\Xi}=\left(\begin{array}{cc}
0 & -\frac{1}{4}\left(\overline{\mathcal{D}}^{2}-4 R\right) \Xi \\
-\frac{1}{4}\left(\mathcal{D}^{2}-4 \bar{R}\right) \Xi & 0
\end{array}\right) .
$$

The operator $\boldsymbol{H}_{\Xi}$ is defined to act on the space of column-vectors

$$
\left(\begin{array}{l}
\eta \\
\bar{\eta}
\end{array}\right), \quad \overline{\mathcal{D}}^{\dot{\alpha}} \eta=0
$$

such that

$$
\boldsymbol{H}_{\Xi}\left(\begin{array}{c}
\eta \\
\bar{\eta}
\end{array}\right)=\left(\begin{array}{c}
-\frac{1}{4}\left(\overline{\mathcal{D}}^{2}-4 R\right)(\Xi \bar{\eta}) \\
-\frac{1}{4}\left(\mathcal{D}^{2}-4 \bar{R}\right)(\Xi \eta)
\end{array}\right) \equiv\left(\begin{array}{c}
\mathcal{P}_{+-}^{\Xi} \bar{\eta} \\
\mathcal{P}_{-+}^{\Xi} \eta
\end{array}\right) .
$$

The quantisation procedure described leads to the following representation for the effective action

$$
\mathrm{e}^{\mathrm{i} \Gamma_{\mathrm{v}}[\Phi, \bar{\Phi}]}=\operatorname{Det} H^{(R)} \operatorname{Det}^{1 / 2} \boldsymbol{H}_{\Xi} \int \mathrm{D} V \exp \left\{-\frac{\mathrm{i}}{2} \int \mathrm{d}^{4} x \mathrm{~d}^{2} \theta \mathrm{d}^{2} \bar{\theta} E V \boldsymbol{\Delta}_{\mathrm{v}} V\right\},
$$

and therefore

$$
\Gamma_{\mathrm{v}}[\Phi, \bar{\Phi}]=\frac{\mathrm{i}}{2} \operatorname{Tr} \ln \boldsymbol{\Delta}_{\mathrm{v}}-\mathrm{i} \operatorname{Tr} \ln H^{(R)}-\frac{\mathrm{i}}{2} \operatorname{Tr} \ln \boldsymbol{H}_{\Xi}
$$

or equivalently

$$
\Gamma_{\mathrm{v}}[\Phi, \bar{\Phi}]=\Gamma_{\mathrm{v}}+\frac{\mathrm{i}}{2} \operatorname{Tr} \ln \frac{\boldsymbol{\Delta}_{\mathrm{v}}}{\square_{\mathrm{v}}}-\frac{\mathrm{i}}{2} \operatorname{Tr} \ln \frac{\boldsymbol{H}_{\Xi}}{H^{(0)}} .
$$

Here $\Gamma_{\mathrm{v}}$ is the effective action for the vector multiplet in a supergravity background [34, 46]. It is obtained from $\Gamma_{\mathrm{v}}[\Phi, \bar{\Phi}]$ by setting $\Phi=\mathrm{i}$. The functional $\Gamma_{\mathrm{v}}$ was studied in [34, 46]. It is the second and third terms in (4.17b) which contain the dependence of $\Gamma_{\mathrm{v}}[\Phi, \bar{\Phi}]$ on $\Phi$ and $\bar{\Phi}$. The latter functionals will be studied in the next sections. 


\section{$5 \quad$ Heat kernel calculations (I)}

Heat kernel techniques in curved superspace have been developed by many authors over several decades [43, 44, 46, 59-64], see [34] for a review. What is special about the contributions in the second and third terms of $(4.17 \mathrm{~b})$, is that they involve non-minimal second-order differential operators for which the standard superfield Schwinger-DeWitt technique $[34,46]$ is not directly applicable.

\subsection{Generalised Schwinger-DeWitt representation}

Associated with a second-order differential operator of the form

$$
\Delta=\Omega^{A B} \mathcal{D}_{B} \mathcal{D}_{A}+\Theta^{A} \mathcal{D}_{A}+\kappa=\hat{\Delta}+\kappa, \quad \Omega^{A B}=(-1)^{\epsilon_{A} \epsilon_{B}} \Omega^{B A}
$$

is the heat kernel $U\left(z, z^{\prime} \mid s\right)$ which satisfies the equation

$$
\left(\mathrm{i} \frac{\partial}{\partial s}+\Delta\right) U\left(z, z^{\prime} \mid s\right)=0
$$

and the initial condition

$$
\lim _{s \rightarrow 0} U\left(z, z^{\prime} \mid s\right)=E^{-1} \delta^{4}\left(x-x^{\prime}\right) \delta^{2}\left(\theta-\theta^{\prime}\right) \delta^{2}\left(\bar{\theta}-\bar{\theta}^{\prime}\right) \equiv \delta^{4 \mid 4}\left(z, z^{\prime}\right) .
$$

Keeping in mind the structure of the operator $\boldsymbol{\Delta}_{\mathrm{v}}$, eq. (4.8), it will be assumed that

$$
\Omega^{a b}=\Omega(z) \eta^{a b}
$$

with $\eta_{a b}$ the Minkowski metric and $\Omega$ a nowhere vanishing background real superfield.

We look for a solution to (5.2) by making the ansatz

$$
U\left(z, z^{\prime} \mid s\right)=-\frac{\mathrm{i}}{(4 \pi s)^{2}} \mathrm{e}^{\mathrm{i} \sigma\left(z, z^{\prime}\right) / 2 s} \sum_{n=0}^{\infty} a_{n}\left(z, z^{\prime}\right)(\mathrm{i} s)^{n},
$$

where the symmetric bi-scalars $\sigma$ and $a_{n}$ obey the equations

$$
\begin{aligned}
\Omega^{A B} \mathcal{D}_{B} \sigma \mathcal{D}_{A} \sigma & =2 \sigma, \\
\Omega^{A B} \mathcal{D}_{B} \sigma \mathcal{D}_{A} a_{0}+\frac{1}{2}(\hat{\Delta} \sigma-4) a_{0} & =0, \\
n a_{n}+\Omega^{A B} \mathcal{D}_{B} \sigma \mathcal{D}_{A} a_{n}+\frac{1}{2}(\hat{\Delta} \sigma-4) a_{n} & =\Delta a_{n-1}, \quad n>0 .
\end{aligned}
$$

The bi-scalars $\sigma$ and $a_{0}$ should obey certain boundary conditions in order ensure the initial condition (5.2b), including the following:

$$
\begin{array}{rlrl}
\sigma(z, z) & =0, & \left.\mathcal{D}_{A} \sigma\left(z, z^{\prime}\right)\right|_{z=z^{\prime}} & =0 ; \\
a_{0}(z, z) & =0, & & \\
\left.\mathcal{D}_{A} a_{0}\left(z, z^{\prime}\right)\right|_{z=z^{\prime}} & =0, & \left.\mathcal{D}^{2} \overline{\mathcal{D}}^{2} a_{0} \sigma\left(z, z^{\prime}\right)\right|_{z=z^{\prime}}=16 .
\end{array}
$$


Here we do not give the complete list of boundary conditions. The important for our analysis result, which follows from the above relations, is the coincidence limit

$$
U(z, z \mid s)=-\frac{\mathrm{i}}{(4 \pi s)^{2}} \sum_{n=2}^{\infty} a_{n}(z, z)(\mathrm{i} s)^{n} .
$$

It is the coefficient $a_{2}(z, z)$ which contributes to the logarithmically divergent part of the effective action. In the case that $\Delta$ coincides with the operator (4.8), the corresponding kernel (5.4) will be denoted $\boldsymbol{U}_{\mathrm{v}}(z, z \mid s)$, and boldface notation will also be used for the bi-scalars in the right-hand side of (5.4).

A typical contribution to the effective action is $\Gamma=\frac{i}{2} \operatorname{Tr} \ln \Delta$, and this can be regularised as

$$
\Gamma_{\omega}=\frac{1}{2} \mu^{2 \omega} \int_{0}^{\infty} \frac{\mathrm{d} s}{(\mathrm{i} s)^{1-\omega}} \int \mathrm{d}^{4} x \mathrm{~d}^{2} \theta \mathrm{d}^{2} \bar{\theta} E U(z, z \mid s)
$$

with $\omega$ the regularisation parameter. In the limit $\omega \rightarrow 0$, one obtains

$$
\Gamma_{\omega}=\frac{1}{32 \pi^{2} \omega} \int \mathrm{d}^{4} x \mathrm{~d}^{2} \theta \mathrm{d}^{2} \bar{\theta} E a_{2}(z, z)+\text { finite part } .
$$

\subsection{Evaluation of the heat kernel in flat superspace}

To compute the $\Xi$-dependent contributions from the second term in (4.17b), it suffices to work in flat superspace and analyse the heat kernel

$$
\boldsymbol{U}_{\mathrm{v}}\left(z, z^{\prime} \mid s\right)=\mathrm{e}^{\mathrm{i} s \boldsymbol{\Delta}_{\mathrm{v}}} \delta^{4 \mid 4}\left(z, z^{\prime}\right), \quad \delta^{4 \mid 4}\left(z, z^{\prime}\right)=\delta^{4}\left(x-x^{\prime}\right) \delta^{2}\left(\theta-\theta^{\prime}\right) \delta^{2}\left(\bar{\theta}-\bar{\theta}^{\prime}\right)
$$

of the operator

$$
\begin{aligned}
\boldsymbol{\Delta}_{\mathrm{v}}= & \Xi \partial^{a} \partial_{a}-\frac{\mathrm{i}}{2}\left(\left(D^{\alpha} \Xi\right) \partial_{\alpha \dot{\alpha}} \bar{D}^{\dot{\alpha}}+\left(\bar{D}^{\dot{\alpha}} \Xi\right) \partial_{\alpha \dot{\alpha}} D^{\alpha}\right)+\frac{1}{16}\left(\left(\bar{D}^{2} \Xi\right) D^{2}+\left(D^{2} \Xi\right) \bar{D}^{2}\right) \\
& -\frac{1}{4} \Xi G^{\alpha \dot{\alpha}}\left[D_{\alpha}, \bar{D}_{\dot{\alpha}}\right]
\end{aligned}
$$

for some real vector superfield $G_{\alpha \dot{\alpha}}$.

When computing the heat kernel in superspace, it is convenient to deal with the supersymmetric interval [58]

$$
\zeta^{A}=\left\{\begin{array}{l}
\zeta^{a}=\left(x-x^{\prime}\right)^{a}-\mathrm{i}\left(\theta-\theta^{\prime}\right) \sigma^{a} \bar{\theta}^{\prime}+\mathrm{i} \theta^{\prime} \sigma^{a}\left(\bar{\theta}-\bar{\theta}^{\prime}\right) \\
\zeta^{\alpha}=\left(\theta-\theta^{\prime}\right)^{\alpha} \\
\bar{\zeta}_{\dot{\alpha}}=\left(\bar{\theta}-\bar{\theta}^{\prime}\right)_{\dot{\alpha}}
\end{array}\right.
$$

We introduce a Fourier transform for the bosonic part of the superspace delta function,

$$
\delta^{4 \mid 4}\left(z, z^{\prime}\right)=\int \frac{\mathrm{d}^{4} k}{(2 \pi)^{4}} \mathrm{e}^{\mathrm{i} k_{a} \zeta^{a}} \zeta^{2} \bar{\zeta}^{2}, \quad \zeta^{2}=\zeta^{\alpha} \zeta_{\alpha}, \quad \bar{\zeta}^{2}=\bar{\zeta}_{\dot{\alpha}} \bar{\zeta}^{\dot{\alpha}}
$$

Making use of this representation, for the heat kernel (5.10) at coincident points we obtain

$$
\begin{aligned}
\boldsymbol{U}_{\mathrm{v}}(z, z \mid s)= & \int \frac{\mathrm{d}^{4} k}{(2 \pi)^{4}} \exp \left\{\mathrm { i } s \left(\Xi X^{a} X_{a}-\frac{\mathrm{i}}{2}\left(D^{\alpha} \Xi X_{\alpha \dot{\alpha}} \bar{X}^{\dot{\alpha}}+\bar{D}^{\dot{\alpha}} \Xi X_{\alpha \dot{\alpha}} X^{\alpha}\right)\right.\right. \\
& \left.\left.+\frac{1}{16}\left(\bar{D}^{2} \Xi X^{\alpha} X_{\alpha}+D^{2} \Xi \bar{X}_{\dot{\alpha}} \bar{X}^{\dot{\alpha}}\right)-\frac{1}{4} \Xi G^{\alpha \dot{\alpha}}\left[X_{\alpha}, \bar{X}_{\dot{\alpha}}\right]\right)\right\}\left.\zeta^{2} \bar{\zeta}^{2}\right|_{\zeta=0},
\end{aligned}
$$


where we have denoted

$$
X_{a}=\partial_{a}+\mathrm{i} k_{a}, \quad X_{\alpha}=D_{\alpha}-k^{a}\left(\sigma_{a}\right)_{\alpha \dot{\alpha}} \bar{\zeta}^{\dot{\alpha}}, \quad \bar{X}^{\dot{\alpha}}=\bar{D}^{\dot{\alpha}}-k^{a}\left(\tilde{\sigma}_{a}\right)^{\dot{\alpha} \alpha} \zeta_{\alpha} .
$$

As usual, it is useful to rescale, $k_{a} \rightarrow s^{-1 / 2} k_{a}$, the integration variable in (5.13). Then it follows from (5.13) that $\boldsymbol{U}_{\mathrm{v}}(z, z \mid s)$ indeed has the asymptotic form (5.7). We denote $\boldsymbol{a}_{n}(z, z)$ the corresponding DeWitt coefficients.

In computing the DeWitt coefficients $\boldsymbol{a}_{n}(z, z)$, the generic term in the Taylor expansion of the right-hand side of (5.13) will involve Gaussian moments of the form

$$
\left\langle k^{a_{1}} \ldots k^{a_{n}}\right\rangle \equiv \frac{1}{\left(4 \pi^{2} s\right)^{2}} \int \mathrm{d}^{4} k \mathrm{e}^{-\mathrm{i} k^{2} \Xi} k^{a_{1}} \ldots k^{a_{n}} .
$$

They can be computed by introducing a generating function $Z(J)$ defined by

$$
Z(J)=\frac{1}{\left(4 \pi^{2} s\right)^{2}} \int \mathrm{d}^{4} k \mathrm{e}^{-\mathrm{i} k^{2} \Xi+J_{a} k^{a}}, \quad\left\langle k^{a_{1}} \ldots k^{a_{n}}\right\rangle=\left.\frac{\partial^{n}}{\partial J_{a_{1}} \ldots \partial J_{a_{n}}} Z(J)\right|_{J=0} .
$$

Then for $Z(J)$ one gets

$$
Z(J)=\frac{\mathrm{i}}{(4 \pi \mathrm{i} s)^{2}} \frac{1}{\Xi^{2}} \exp \left(-\frac{\mathrm{i}}{4 \Xi} J^{2}\right) .
$$

The result of calculation of the coefficient $\boldsymbol{a}_{2}(z, z)$ is

$$
\boldsymbol{a}_{2}(z, z)=\frac{1}{16} \frac{\nabla^{2} \Xi \bar{\nabla}^{2} \Xi-8 G^{\alpha \dot{\alpha}} D_{\alpha} \Xi \bar{D}_{\dot{\alpha}} \Xi}{\Xi^{2}}+\frac{1}{8}(D \ln \Xi)^{2}(\bar{D} \ln \Xi)^{2}-G^{a} G_{a},
$$

where we have denoted

$$
\nabla^{2} \Xi:=D^{2} \Xi-2 \frac{(D \Xi)^{2}}{\Xi} .
$$

The expression (5.18) can now be lifted to curved superspace by replacing $D_{A} \rightarrow \mathcal{D}_{A}$. Recalling the expression for $\Xi$ in terms of $\Phi$ and its conjugate, $\Xi=\mathrm{i}(\bar{\Phi}-\Phi)$, we obtain

$$
\begin{aligned}
\boldsymbol{a}_{2}(z, z)= & -\frac{1}{16} \frac{1}{(\Phi-\bar{\Phi})^{2}}\left\{\nabla^{2} \Phi \bar{\nabla}^{2} \bar{\Phi}-8 \mathcal{D}^{\alpha} \Phi G_{\alpha \dot{\alpha}} \overline{\mathcal{D}}^{\dot{\alpha}} \bar{\Phi}\right\} \\
& +\frac{1}{8} \frac{1}{(\Phi-\bar{\Phi})^{4}} \mathcal{D}^{\alpha} \Phi \mathcal{D}_{\alpha} \Phi \overline{\mathcal{D}}_{\dot{\alpha}} \bar{\Phi} \overline{\mathcal{D}}^{\dot{\alpha}} \bar{\Phi}-G^{a} G_{a},
\end{aligned}
$$

where $\nabla^{2} \Phi$ and $\bar{\nabla}^{2} \bar{\Phi}$ are defined in (2.4). This result can also be derived directly in curved superspace by making use of either (i) the recurrence relations (5.5); or (ii) superspace normal coordinates [65]. The latter approach has recently been advocated in [64].

We note that the $a_{2}$-coefficient for the minimal operator (4.9) was computed in [46]. The result is $a_{2}(z, z)=-G^{a} G_{a}$. Then, the logarithmically divergent contribution from in the second term in $(4.17 \mathrm{~b})$ is determined by

$$
\begin{aligned}
\boldsymbol{a}_{2}(z, z)-a_{2}(z, z)= & -\frac{1}{16} \frac{1}{(\Phi-\bar{\Phi})^{2}}\left\{\nabla^{2} \Phi \bar{\nabla}^{2} \bar{\Phi}-8 \mathcal{D}^{\alpha} \Phi G_{\alpha \dot{\alpha}} \overline{\mathcal{D}}^{\dot{\alpha}} \bar{\Phi}\right\} \\
& +\frac{1}{8} \frac{1}{(\Phi-\bar{\Phi})^{4}} \mathcal{D}^{\alpha} \Phi \mathcal{D}_{\alpha} \Phi \overline{\mathcal{D}}_{\dot{\alpha}} \bar{\Phi} \overline{\mathcal{D}}^{\dot{\alpha}} \bar{\Phi}
\end{aligned}
$$

This contribution is exactly of the type given by (2.3). 


\subsection{Super-Weyl anomaly}

Here we compute the super-Weyl variation of the functional

$$
W^{(\mathrm{v})}=\frac{\mathrm{i}}{2} \operatorname{Tr} \ln \frac{\boldsymbol{\Delta}_{\mathrm{v}}}{\square_{\mathrm{v}}},
$$

which is the second term in (4.17b). We are going to work with its regularised version

$$
W_{\omega}^{(\mathrm{v})}=\frac{1}{2} \mu^{2 \omega} \int_{0}^{\infty} \frac{\mathrm{d} s}{(\mathrm{i} s)^{1-\omega}} \int \mathrm{d}^{4} x \mathrm{~d}^{2} \theta \mathrm{d}^{2} \bar{\theta} E\left\{\boldsymbol{U}_{\mathrm{v}}(z, z \mid s)-U_{\mathrm{v}}(z, z \mid s)\right\},
$$

where $U_{\mathrm{v}}(s)$ is the heat kernel associated with the operator (4.9). The super-Weyl variation of $\boldsymbol{\Delta}_{\mathrm{V}}$ is readily determined by representing this operator as

$$
\begin{aligned}
\boldsymbol{\Delta}_{\mathrm{v}} & =\frac{\mathrm{i}}{8} \mathcal{D}^{\alpha} \Phi\left(\overline{\mathcal{D}}^{2}-4 R\right) \mathcal{D}_{\alpha}-\frac{\mathrm{i}}{8} \overline{\mathcal{D}}_{\dot{\alpha}} \bar{\Phi}\left(\mathcal{D}^{2}-4 \bar{R}\right) \overline{\mathcal{D}}^{\dot{\alpha}}+\mathcal{P}_{+-} \Xi \mathcal{P}_{-+}+\mathcal{P}_{-+} \Xi \mathcal{P}_{+-}, \\
\mathcal{P}_{+-} & :=-\frac{1}{4}\left(\overline{\mathcal{D}}^{2}-4 R\right), \quad \mathcal{P}_{-+}:=-\frac{1}{4}\left(\mathcal{D}^{2}-4 \bar{R}\right)
\end{aligned}
$$

where $\Phi, \bar{\Phi}$ and $\Xi$ are viewed as operators. With the understanding that $\boldsymbol{\Delta}_{\mathrm{v}}$ acts on the space of scalar superfields, the super-Weyl variation of $\boldsymbol{\Delta}_{\mathrm{v}}$ can be represented as

$$
\begin{aligned}
\delta_{\sigma} \boldsymbol{\Delta}_{\mathrm{v}}= & (\sigma+\bar{\sigma}) \boldsymbol{\Delta}_{\mathrm{v}}+(\sigma-\bar{\sigma})\left(\mathcal{P}_{+-} \Xi \mathcal{P}_{-+}-\mathcal{P}_{-+} \Xi \mathcal{P}_{+-}\right) \\
& -\left(\mathcal{P}_{+-} \Xi \mathcal{P}_{-+}-\mathcal{P}_{-+} \Xi \mathcal{P}_{+-}\right)(\sigma-\bar{\sigma}) .
\end{aligned}
$$

The super-Weyl transformation of $\square_{\mathrm{V}}$ is obtained from (5.25) by replacing $\boldsymbol{\Delta}_{\mathrm{v}} \rightarrow \square_{\mathrm{V}}$ and setting $\Xi=1$. These transformations and certain Ward identities, similar to those described in appendix $\mathrm{C}$ of [46], should be used to derive the super-Weyl variation of (5.23). The result is

$$
\delta_{\sigma} W_{\omega}^{(\mathrm{v})}=-\frac{1}{2} \omega \int_{0}^{\infty} \frac{\mu^{2 \omega} \mathrm{d} s}{(\mathrm{i} s)^{1-\omega}} \int \mathrm{d}^{4} x \mathrm{~d}^{2} \theta \mathrm{d}^{2} \bar{\theta} E(\sigma+\bar{\sigma})\left\{\boldsymbol{U}_{\mathrm{v}}(z, z \mid s)-U_{\mathrm{v}}(z, z \mid s)\right\} .
$$

In the limit $\omega \rightarrow 0$ we obtain

$$
\begin{aligned}
\delta_{\sigma} W_{\text {ren }}^{(\mathrm{v})}=-\frac{1}{32 \pi^{2}} \int \mathrm{d}^{4} x \mathrm{~d}^{2} \theta \mathrm{d}^{2} \bar{\theta} E(\sigma+\bar{\sigma})\left\{\boldsymbol{a}_{2}(z, z)-a_{2}(z, z)\right\} \\
=\frac{1}{512 \pi^{2}} \int \mathrm{d}^{4} x \mathrm{~d}^{2} \theta \mathrm{d}^{2} \bar{\theta} E(\sigma+\bar{\sigma})\left\{\frac{1}{(\Phi-\bar{\Phi})^{2}}\left(\nabla^{2} \Phi \bar{\nabla}^{2} \bar{\Phi}-8 \mathcal{D}^{\alpha} \Phi G_{\alpha \dot{\alpha}} \overline{\mathcal{D}}^{\dot{\alpha}} \bar{\Phi}\right)\right. \\
\left.-2 \frac{1}{(\Phi-\bar{\Phi})^{4}} \mathcal{D}^{\alpha} \Phi \mathcal{D}_{\alpha} \Phi \overline{\mathcal{D}}_{\dot{\alpha}} \bar{\Phi} \overline{\mathcal{D}}^{\dot{\alpha}} \bar{\Phi}\right\}
\end{aligned}
$$

\section{$6 \quad$ Heat kernel calculations (II)}

Now we turn to computing the contribution from the third term in (4.17b). For this we first need to analyse the effective action of the following model in curved superspace

$$
S[\Psi, \bar{\Psi} ; \mathcal{V}]=\int \mathrm{d}^{4} x \mathrm{~d}^{2} \theta \mathrm{d}^{2} \bar{\theta} E \bar{\Psi} \mathrm{e}^{\mathcal{V}} \Psi, \quad \overline{\mathcal{D}}^{\dot{\alpha}} \Psi=0 .
$$


Here the dynamical variables are the chiral scalar superfield $\Psi$ and its conjugate $\bar{\Psi}$. They couple to a background scalar superfield $\mathcal{V}$. At the classical level, the action (6.1) possesses two local symmetries associated with the background fields: (i) it is invariant under gauge transformations

$$
\delta_{\lambda} \mathcal{V}=\lambda+\bar{\lambda}, \quad \delta_{\lambda} \Psi=-\lambda \Psi, \quad \overline{\mathcal{D}}^{\dot{\alpha}} \lambda=0
$$

(ii) it is invariant under super-Weyl transformations acting on $\Psi$ and $V$ as follows:

$$
\delta_{\sigma} \Psi=\sigma \Psi, \quad \delta_{\sigma} V=0 .
$$

Both symmetries are anomalous at the quantum level. The anomalies were computed in $[46,61]$, and our discussion here will build on the results of these publications.

Effective action $\Gamma[\Xi]$ is defined by

$$
\mathrm{e}^{\mathrm{i} \Gamma[\Xi]}=\int \mathrm{D} \bar{\Psi} \mathrm{D} \Psi \exp (\mathrm{i} S[\Psi, \bar{\Psi} ; \mathcal{V}]), \quad \Xi:=\mathrm{e}^{\mathcal{V}}
$$

and can be expressed as

$$
\Gamma[\Xi]=\frac{\mathrm{i}}{2} \operatorname{Tr} \ln H_{\Xi}=\frac{\mathrm{i}}{4} \operatorname{Tr}_{+} \ln \left(\mathcal{P}_{+-}^{\Xi} \mathcal{P}_{-+}^{\Xi}\right)+\frac{\mathrm{i}}{4} \operatorname{Tr}_{-} \ln \left(\mathcal{P}_{-+}^{\Xi} \mathcal{P}_{+-}^{\Xi}\right) .
$$

The operators $H_{\Xi}$ and $\mathcal{P}_{+-}^{\Xi}, \mathcal{P}_{-+}^{\Xi}$ are defined in (4.14) and (4.15), respectively. In the right-hand side of (6.5), $\operatorname{Tr}_{+}$denotes the chiral trace,

$$
\operatorname{Tr}_{+} A=\int \mathrm{d}^{4} x \mathrm{~d}^{2} \theta \mathcal{E} A(z, z), \quad A\left(z, z^{\prime}\right):=A \delta_{+}\left(z, z^{\prime}\right) .
$$

Here $A$ is an operator acting on the space of covariantly chiral scalar superfields, and $\delta_{+}\left(z, z^{\prime}\right)$ is the chiral delta-function defined by $(6.10)$.

\subsection{Generalised Schwinger-DeWitt representation}

As follows from the representation (6.5), $\Gamma[\Xi]$ can be expressed in terms of the heat kernels $\boldsymbol{U}_{\mathrm{c}}\left(z, z^{\prime} \mid s\right)$ and $\boldsymbol{U}_{\mathrm{a}}\left(z, z^{\prime} \mid s\right)$ of the chiral $\left(\boldsymbol{\Delta}_{\mathrm{c}}\right)$ and antichiral $\left(\boldsymbol{\Delta}_{\mathrm{a}}\right)$ operators, respectively, which are defined as

$$
\begin{aligned}
& \boldsymbol{\Delta}_{\mathrm{c}}:=\mathcal{P}_{+-}^{\Xi} \mathcal{P}_{-+}^{\Xi}=\frac{1}{16}\left(\overline{\mathcal{D}}^{2}-4 R\right) \Xi\left(\mathcal{D}^{2}-4 \bar{R}\right) \Xi, \\
& \boldsymbol{\Delta}_{\mathrm{a}}:=\mathcal{P}_{-+}^{\Xi} \mathcal{P}_{+-}^{\Xi}=\frac{1}{16}\left(\mathcal{D}^{2}-4 \bar{R}\right) \Xi\left(\overline{\mathcal{D}}^{2}-4 R\right) \Xi .
\end{aligned}
$$

The action of $\boldsymbol{\Delta}_{\mathrm{c}}$ on a covariantly chiral scalar $\eta, \overline{\mathcal{D}}^{\dot{\alpha}} \eta=0$, is given by

$$
\begin{aligned}
\boldsymbol{\Delta}_{\mathrm{c}} \eta= & \left(\Xi^{2} \mathcal{D}^{a} \mathcal{D}_{a}-\frac{\mathrm{i}}{4}\left(\overline{\mathcal{D}}_{\dot{\alpha}} \Xi^{2}\right)\left\{\mathcal{D}^{\alpha \dot{\alpha}}, \mathcal{D}_{\alpha}\right\}+\frac{1}{16}\left[\left(\overline{\mathcal{D}}^{2}+4 R\right) \Xi^{2}\right] \mathcal{D}^{2}\right. \\
& -\frac{\mathrm{i}}{2}\left[\Xi^{2} G^{\alpha \dot{\alpha}}+\frac{1}{2}\left(\overline{\mathcal{D}}^{\dot{\alpha}} \mathcal{D}^{\alpha} \Xi^{2}\right)\right] \mathcal{D}_{\alpha \dot{\alpha}}+\frac{1}{4}\left[\Xi^{2}\left(\mathcal{D}^{\alpha} R\right)+\frac{1}{4}\left(\overline{\mathcal{D}}^{2} \mathcal{D}^{\alpha} \Xi^{2}\right)\right] \mathcal{D}_{\alpha} \\
& \left.+\frac{1}{16}\left(\overline{\mathcal{D}}^{2}-4 R\right)\left[\Xi\left(\mathcal{D}^{2}-4 \bar{R}\right) \Xi\right]\right) \eta, \quad \overline{\mathcal{D}}^{\dot{\alpha}} \boldsymbol{\Delta}_{\mathrm{c}} \eta=0 .
\end{aligned}
$$


The chirality of $\boldsymbol{\Delta}_{\mathrm{c}} \eta$ implies the existence of a symmetric bracket, $\Psi_{1} \star \Psi_{2}=\Psi_{2} \star \Psi_{1}$, on the space of covariantly chiral scalars:

$$
\begin{aligned}
\Psi_{1} \star \Psi_{2}:= & \Xi^{2} \mathcal{D}^{a} \Psi_{1} \mathcal{D}_{a} \Psi_{2}-\frac{\mathrm{i}}{4}\left(\overline{\mathcal{D}}_{\dot{\alpha}} \Xi^{2}\right)\left(\mathcal{D}^{\alpha \dot{\alpha}} \Psi_{1} \mathcal{D}_{\alpha} \Psi_{2}+\mathcal{D}^{\alpha \dot{\alpha}} \Psi_{2} \mathcal{D}_{\alpha} \Psi_{1}\right) \\
& +\frac{1}{16}\left(\left(\overline{\mathcal{D}}^{2}+4 R\right) \Xi^{2}\right) \mathcal{D}^{\alpha} \Psi_{1} \mathcal{D}_{\alpha} \Psi_{2}
\end{aligned}
$$

For arbitrary chiral scalars $\Psi_{1}$ and $\Psi_{2}$, their bracket $\Psi_{1} \star \Psi_{2}$ is chiral. This may be checked using the algebra of covariant derivatives (A.2). Setting $\Xi=1$ in (6.9) gives the bracket introduced in [34, 46].

It should be remarked that both operators (4.8) and (6.8) are non-minimal. Instead of dealing with $\boldsymbol{\Delta}_{\mathrm{v}}$, one can equivalently work with the minimal symmetric operator $\widetilde{\boldsymbol{\Delta}}_{\mathrm{v}}=$ $\Xi^{-\frac{1}{2}} \boldsymbol{\Delta}_{\mathrm{v}} \Xi^{-\frac{1}{2}}$, which is of the type studied in $[34,46] .{ }^{9}$ However, such a transformation is not possible for the chiral operator $\boldsymbol{\Delta}_{\mathrm{c}}$, eq. (6.8).

Let us introduce the heat kernel associated with the chiral operator $\boldsymbol{\Delta}_{\mathrm{c}}$,

$$
\boldsymbol{U}_{\mathrm{c}}\left(z, z^{\prime} \mid s\right)=\mathrm{e}^{\mathrm{i} s \boldsymbol{\Delta}_{\mathrm{c}}} \delta_{+}\left(z, z^{\prime}\right), \quad \delta_{+}\left(z, z^{\prime}\right)=-\frac{1}{4}\left(\overline{\mathcal{D}}^{2}-4 R\right) \delta^{4 \mid 4}\left(z, z^{\prime}\right)
$$

The heat kernel is chiral in both superspace argument $z$ and $z^{\prime}$. By construction, it obeys the equation

$$
\left(\mathrm{i} \frac{\partial}{\partial s}+\boldsymbol{\Delta}_{\mathrm{c}}\right) \boldsymbol{U}_{\mathrm{c}}\left(z, z^{\prime} \mid s\right)=0
$$

and the initial condition

$$
\boldsymbol{U}_{\mathrm{c}}\left(z, z^{\prime} \mid s \rightarrow 0\right)=\delta_{+}\left(z, z^{\prime}\right)
$$

We look for a solution to (6.11) by making the ansatz

$$
\boldsymbol{U}_{\mathrm{c}}\left(z, z^{\prime} \mid s\right)=-\frac{\mathrm{i}}{(4 \pi s)^{2}} \mathrm{e}^{\mathrm{i} \boldsymbol{\sigma}_{\mathrm{c}}\left(z, z^{\prime}\right) / 2 s} \sum_{n=0}^{\infty} \boldsymbol{a}_{n}^{\mathrm{c}}\left(z, z^{\prime}\right)(\mathrm{i} s)^{n} .
$$

Here the symmetric bi-scalars $\boldsymbol{\sigma}$ and $\boldsymbol{a}_{n}$ are covariantly chiral and obey the equations

$$
\begin{aligned}
\boldsymbol{\sigma}_{\mathrm{c}} \star \boldsymbol{\sigma}_{\mathrm{c}} & =2 \boldsymbol{\sigma}_{\mathrm{c}}, \\
\boldsymbol{\sigma}_{\mathrm{c}} \star \boldsymbol{a}_{0}^{\mathrm{c}}+\frac{1}{2}\left(\hat{\boldsymbol{\Delta}}_{\mathrm{c}} \boldsymbol{\sigma}_{\mathrm{c}}-4\right) \boldsymbol{a}_{0}^{\mathrm{c}} & =0, \\
n \boldsymbol{a}_{n}^{\mathrm{c}}+\boldsymbol{\sigma}_{\mathrm{c}} \star \boldsymbol{a}_{n}^{\mathrm{c}}+\frac{1}{2}\left(\hat{\boldsymbol{\Delta}}_{\mathrm{c}} \boldsymbol{\sigma}_{\mathrm{c}}-4\right) \boldsymbol{a}_{n}^{\mathrm{c}} & =\boldsymbol{\Delta}_{\mathrm{c}} \boldsymbol{a}_{n-1}, \quad n>0,
\end{aligned}
$$

where we have introduced the differential opeartor

$$
\hat{\boldsymbol{\Delta}}_{\mathrm{c}}=\boldsymbol{\Delta}_{\mathrm{c}}-\frac{1}{16}\left(\overline{\mathcal{D}}^{2}-4 R\right)\left[\Xi\left(\mathcal{D}^{2}-4 \bar{R}\right) \Xi\right] .
$$

\footnotetext{
${ }^{9} \mathrm{~A}$ similar approach was pursued by Osborn in the non-supersymmetric case [19].
} 
The bi-scalars $\boldsymbol{\sigma}_{\mathrm{c}}$ and $\boldsymbol{a}_{0}^{\mathrm{c}}$ should obey certain boundary conditions in order ensure the initial condition $(5.2 \mathrm{~b})$, including the following:

$$
\begin{array}{lll}
\boldsymbol{\sigma}_{\mathrm{c}}(z, z)=0, & \left.\mathcal{D}_{A} \boldsymbol{\sigma}_{\mathrm{c}}\left(z, z^{\prime}\right)\right|_{z=z^{\prime}}=0 ; \\
\boldsymbol{a}_{0}^{\mathrm{c}}(z, z)=0, & \left.\mathcal{D}_{A} \boldsymbol{a}_{0}^{\mathrm{c}}\left(z, z^{\prime}\right)\right|_{z=z^{\prime}}=0, & \left.\mathcal{D}^{2} \boldsymbol{a}_{0}^{\mathrm{c}} \sigma\left(z, z^{\prime}\right)\right|_{z=z^{\prime}}=-4 .
\end{array}
$$

The complete set of boundary condition in the $\Xi=1$ case is given in [34, 46].

The heat kernel at coincident points is

$$
\boldsymbol{U}_{\mathrm{c}}(z, z \mid s)=-\frac{\mathrm{i}}{(4 \pi s)^{2}} \sum_{n=1}^{\infty} \boldsymbol{a}_{n}^{\mathrm{c}}(z, z)(\mathrm{i} s)^{n} .
$$

It is $\boldsymbol{U}_{\mathrm{c}}(z, z \mid s)$ and its antichiral twin $\boldsymbol{U}_{\mathrm{a}}(z, z \mid s)$ which determine the regularised effective action

$$
\begin{aligned}
\Gamma_{\omega}[\Xi] & =\frac{1}{4} \mu^{2 \omega} \int_{0}^{\infty} \frac{\mathrm{d} s}{(\mathrm{i} s)^{1-\omega}}\left\{\operatorname{Tr}_{+} \boldsymbol{U}_{\mathrm{c}}(s)+\operatorname{Tr}_{-} \boldsymbol{U}_{\mathrm{a}}(s)\right\} \\
& =\frac{1}{4} \mu^{2 \omega} \int_{0}^{\infty} \frac{\mathrm{d} s}{(\mathrm{i} s)^{1-\omega}} \int \mathrm{d}^{4} x \mathrm{~d}^{2} \theta \mathcal{E} \boldsymbol{U}_{\mathrm{c}}(z, z \mid s)+\text { antichiral } .
\end{aligned}
$$

In order to derive the asymptotic expansion (6.19), one does not need to assume that $\boldsymbol{U}_{\mathrm{c}}\left(z, z^{\prime} \mid s\right)$ has the form (6.13). It suffices to start from the definition (6.10) and then make use of the superspace normal coordinates [65] in order to carry out calculations similar to those employed in [43, 44, 62-64].

The DeWitt coefficient $\boldsymbol{a}_{2}^{\mathrm{c}}(z, z)$ was computed in [61]. It is given by

$$
\begin{aligned}
\boldsymbol{a}_{2}^{\mathrm{c}}(z, z)= & \frac{1}{4} \mathcal{W}^{\alpha} \mathcal{W}_{\alpha}+\frac{1}{12} W^{\alpha \beta \gamma} W_{\alpha \beta \gamma}+\frac{1}{48}\left(\overline{\mathcal{D}}^{2}-4 R\right)\left(G^{a} G_{a}+2 R \bar{R}\right) \\
& -\frac{1}{96}\left(\overline{\mathcal{D}}^{2}-4 R\right) \mathcal{D}^{2} R
\end{aligned}
$$

where

$$
\mathcal{W}_{\alpha}=-\frac{1}{4}\left(\overline{\mathcal{D}}^{2}-4 R\right) \mathcal{D}_{\alpha} \mathcal{V}=-\frac{1}{4}\left(\overline{\mathcal{D}}^{2}-4 R\right) \mathcal{D}_{\alpha} \ln \Xi .
$$

Setting $\mathcal{W}_{\alpha}=0$ in (6.21a) gives the DeWitt coefficient $a_{2}^{\mathrm{c}}(z, z)$ corresponding to the chiral d'Alembertian

$$
\begin{aligned}
\square_{\mathrm{c}} & =\frac{1}{16}\left(\overline{\mathcal{D}}^{2}-4 R\right)\left(\mathcal{D}^{2}-4 \bar{R}\right) \\
\square_{\mathrm{c}} \eta & =\left\{\mathcal{D}^{a} \mathcal{D}_{a}+\frac{1}{4} R \mathcal{D}^{2}+\mathrm{i} G^{a} \mathcal{D}_{a}+\frac{1}{4}\left(\mathcal{D}^{\alpha} R\right) \mathcal{D}_{\alpha}-\frac{1}{4}\left[\left(\overline{\mathcal{D}}^{2}-4 R\right) \bar{R}\right]\right\} \eta,
\end{aligned}
$$

with $\eta$ being a chiral scalar superfield. The coefficient $a_{2}^{\mathrm{c}}(z, z)$ was computed for the first time in $[43,44]$ and then re-derived in [46] using an alternative technique.

Now, the logarithmically divergent contribution from in the third term in $(4.17 \mathrm{~b})$ is determined by the chiral operator

$$
\boldsymbol{a}_{2}^{\mathrm{c}}(z, z)-a_{2}^{\mathrm{c}}(z, z)=\frac{1}{4} \mathcal{W}^{\alpha} \mathcal{W}_{\alpha}
$$


and its conjugate. One may check that

$$
\begin{aligned}
\int \mathrm{d}^{4} x \mathrm{~d}^{2} \theta \mathcal{E} \mathcal{W}^{\alpha} \mathcal{W}_{\alpha} & =\frac{1}{4} \int \mathrm{d}^{4} x \mathrm{~d}^{2} \theta \mathrm{d}^{2} \bar{\theta} E \mathcal{D}^{\alpha} \ln \Xi \mathcal{D}_{\alpha} \ln \Xi \overline{\mathcal{D}}_{\dot{\alpha}} \ln \Xi \overline{\mathcal{D}}^{\dot{\alpha}} \ln \Xi \\
& =\frac{1}{4} \int \mathrm{d}^{4} x \mathrm{~d}^{2} \theta \mathrm{d}^{2} \bar{\theta} E \frac{1}{(\Phi-\bar{\Phi})^{4}} \mathcal{D}^{\alpha} \Phi \mathcal{D}_{\alpha} \Phi \overline{\mathcal{D}}_{\dot{\alpha}} \bar{\Phi} \overline{\mathcal{D}}^{\dot{\alpha}} \bar{\Phi}
\end{aligned}
$$

\subsection{Chiral and super-Weyl anomalies}

Here we briefly re-derive the chiral and super-Weyl anomalies in the model (6.1) following $[46,61]$.

Under the chiral transformation (6.2), the operator $\mathcal{P}_{+-}^{\Xi}$ defined by $(4.15)$ varies by the rule

$$
\delta_{\lambda} \mathcal{P}_{+-}^{\Xi}=\mathcal{P}_{+-}^{\Xi}(\lambda+\bar{\lambda})=\lambda \mathcal{P}_{+-}^{\Xi}+\mathcal{P}_{+-}^{\Xi} \bar{\lambda} .
$$

Then making use of (6.7) and (6.10) leads to

$$
\delta_{\lambda} \operatorname{Tr}_{+} \boldsymbol{U}_{\mathrm{c}}(s)=2 s \frac{\partial}{\partial s} \operatorname{Tr}_{+}\left(\lambda \boldsymbol{U}_{\mathrm{c}}(s)\right)+2 \mathrm{i} s \operatorname{Tr}_{-}\left(\bar{\lambda} \mathcal{P}_{-+}^{\Xi} \boldsymbol{U}_{\mathrm{c}}(s) \mathcal{P}_{+-}^{\Xi}\right) .
$$

Due to the identity $\boldsymbol{U}_{\mathrm{c}}(s) \mathcal{P}_{+-}^{\Xi}=\mathcal{P}_{+}^{\Xi} \boldsymbol{U}_{\mathrm{a}}(s)$, we obtain

$$
\delta_{\lambda} \operatorname{Tr}_{+} \boldsymbol{U}_{\mathrm{c}}(s)=2 s \frac{\partial}{\partial s}\left\{\operatorname{Tr}_{+}\left(\lambda \boldsymbol{U}_{\mathrm{c}}(s)\right)+\operatorname{Tr}_{-}\left(\bar{\lambda} \boldsymbol{U}_{\mathrm{a}}(s)\right)\right\} .
$$

From here we can read off the chiral anomaly

$$
\delta_{\lambda} \Gamma_{\text {ren }}[\Xi]=-\frac{1}{16 \pi^{2}} \int \mathrm{d}^{4} x \mathrm{~d}^{2} \theta \mathcal{E} \lambda \boldsymbol{a}_{2}^{\mathrm{c}}(z, z)+\text { c.c. },
$$

where $\Gamma_{\text {ren }}[\Xi]$ stands for the renormalised effective action. In the flat superspace limit (6.28) reduces to the results obtained by Clark, Piguet and Sibold [66] more than forty years ago.

Analogous calculations can be used to compute the super-Weyl anomaly. An infinitesimal super-Weyl transformation acts on $\mathcal{P}_{+-}^{\Xi}$ as

$$
\delta_{\sigma} \mathcal{P}_{+-}^{\Xi}=2 \sigma \mathcal{P}_{+-}^{\Xi}-\mathcal{P}_{+-}^{\Xi} \bar{\sigma}=\mathcal{P}_{+-}^{\Xi}(2 \sigma-\bar{\sigma}) .
$$

This leads to

$$
\delta_{\sigma} \operatorname{Tr}_{+} \boldsymbol{U}_{\mathrm{c}}(s)=s \frac{\partial}{\partial s}\left\{\operatorname{Tr}_{+}\left(\sigma \boldsymbol{U}_{\mathrm{c}}(s)\right)+\operatorname{Tr}_{-}\left(\bar{\sigma} \boldsymbol{U}_{\mathrm{a}}(s)\right)\right\} .
$$

As a consequence, the super-Weyl variation of the renormalised effective action is

$$
\delta_{\sigma} \Gamma_{\text {ren }}[\Xi]=-\frac{1}{32 \pi^{2}} \int \mathrm{d}^{4} x \mathrm{~d}^{2} \theta \mathcal{E} \sigma \boldsymbol{a}_{2}^{\mathrm{c}}(z, z)+\text { c.c. }
$$

Both anomalies (6.28) and (6.31) are determined by the chiral coefficient $\boldsymbol{a}_{2}^{\mathrm{c}}(z, z)$.

The above results allow us to compute the super-Weyl anomaly corresponding to

$$
W^{(\mathrm{c})}=-\frac{\mathrm{i}}{2} \operatorname{Tr} \ln \frac{\boldsymbol{H}_{\Xi}}{H^{(0)}},
$$


which is the third term in the effective action (4.17b). It follows from (6.31) that

$$
\delta_{\sigma} W_{\text {ren }}^{(\mathrm{c})}=\frac{1}{32 \pi^{2}} \int \mathrm{d}^{4} x \mathrm{~d}^{2} \theta \mathcal{E} \sigma\left\{\boldsymbol{a}_{2}^{\mathrm{c}}(z, z)-a_{2}^{\mathrm{c}}(z, z)\right\}+\text { c.c. }
$$

Making use of the relations (6.23) and (6.24) gives

$$
\delta_{\sigma} W_{\text {ren }}^{(\mathrm{c})}=\frac{1}{512 \pi^{2}} \int \mathrm{d}^{4} x \mathrm{~d}^{2} \theta \mathcal{E}(\sigma+\bar{\sigma}) \frac{1}{(\Phi-\bar{\Phi})^{4}} \mathcal{D}^{\alpha} \Phi \mathcal{D}_{\alpha} \Phi \overline{\mathcal{D}}_{\dot{\alpha}} \bar{\Phi} \overline{\mathcal{D}}^{\dot{\alpha}} \bar{\Phi}
$$

\section{Concluding comments}

We are finally prepared to read off the $\Phi$-dependent sector of the logarithmically divergent part of the effective action (4.17b). It is given by

$$
\begin{aligned}
\left(\Gamma_{\mathrm{v}}[\Phi, \bar{\Phi}]-\Gamma_{\mathrm{v}}\right)_{\mathrm{div}}=\frac{1}{32 \pi^{2} \omega}\{ & \int \mathrm{d}^{4} x \mathrm{~d}^{2} \theta \mathrm{d}^{2} \bar{\theta} E\left(\boldsymbol{a}_{2}(z, z)-a_{2}(z, z)\right) \\
& \left.-\int \mathrm{d}^{4} x \mathrm{~d}^{2} \theta \mathrm{d}^{2} \mathcal{E}\left(\boldsymbol{a}_{2}^{\mathrm{c}}(z, z)-a_{2}^{\mathrm{c}}(z, z)\right)\right\} .
\end{aligned}
$$

Making use of the relations (5.21), (6.23) and (6.24), for the right-hand side we obtain

$$
-\frac{1}{512 \pi^{2} \omega} \int \mathrm{d}^{4} x \mathrm{~d}^{2} \theta \mathrm{d}^{2} \bar{\theta} E\left\{\frac{\nabla^{2} \Phi \bar{\nabla}^{2} \bar{\Phi}-8 \mathcal{D}^{\alpha} \Phi G_{\alpha \dot{\alpha}} \overline{\mathcal{D}}^{\dot{\alpha}} \bar{\Phi}}{(\Phi-\bar{\Phi})^{2}}-\frac{\mathcal{D}^{\alpha} \Phi \mathcal{D}_{\alpha} \Phi \overline{\mathcal{D}}_{\dot{\alpha}} \bar{\Phi} \overline{\mathcal{D}}^{\dot{\alpha}} \bar{\Phi}}{(\Phi-\bar{\Phi})^{4}}\right\}
$$

This induced action is of the form (2.3).

We are also in a position to determine the super-Weyl variation of the renormalised effective action (4.17b). Making use of the relations (5.27) and (6.34) gives

$$
\begin{aligned}
\delta_{\sigma}\left(\Gamma_{\mathrm{v}}[\Phi, \bar{\Phi}]-\Gamma_{\mathrm{v}}\right)_{\mathrm{ren}}= & \frac{1}{512 \pi^{2}} \int \mathrm{d}^{4} x \mathrm{~d}^{2} \theta \mathrm{d}^{2} \bar{\theta} E(\sigma+\bar{\sigma}) \\
& \times\left\{\frac{\nabla^{2} \Phi \bar{\nabla}^{2} \bar{\Phi}-8 \mathcal{D}^{\alpha} \Phi G_{\alpha \dot{\alpha}} \overline{\mathcal{D}}^{\dot{\alpha}} \bar{\Phi}}{(\Phi-\bar{\Phi})^{2}}-\frac{\mathcal{D}^{\alpha} \Phi \mathcal{D}_{\alpha} \Phi \overline{\mathcal{D}}_{\dot{\alpha}} \bar{\Phi} \overline{\mathcal{D}}^{\dot{\alpha}} \bar{\Phi}}{(\Phi-\bar{\Phi})^{4}}\right\}
\end{aligned}
$$

This anomaly is of the form (3.17).

The results of the last three sections can be extended to the case of $n$ vector multiplets, with the duality group $\operatorname{SL}(2, \mathbb{R}) \cong \operatorname{Sp}(2, \mathbb{R})$ being replaced with $\operatorname{Sp}(2 n, \mathbb{R})$. For certain Hermitian symmetric spaces including $\operatorname{Sp}(2, \mathbb{R}) / \mathrm{U}(1)$ and $\mathbb{C} P^{n}$,

$$
R_{I \bar{J} K \bar{L}} \mathcal{D}^{\alpha} \Phi^{I} \mathcal{D}_{\alpha} \Phi^{K} \overline{\mathcal{D}}_{\dot{\alpha}} \bar{\Phi}^{\bar{J}} \overline{\mathcal{D}}^{\dot{\alpha}} \bar{\Phi}^{\bar{L}} \propto g_{I \bar{J}} g_{K \bar{L}} \mathcal{D}^{\alpha} \Phi^{I} \mathcal{D}_{\alpha} \Phi^{K} \overline{\mathcal{D}}_{\dot{\alpha}} \bar{\Phi}^{\bar{J}} \overline{\mathcal{D}}^{\dot{\alpha}} \bar{\Phi}^{\bar{L}}
$$

For a generic Kähler manifold $\mathcal{M}$, however, the last term in (2.6) and the following functional

$$
\int \mathrm{d}^{4} x \mathrm{~d}^{2} \theta \mathrm{d}^{2} \bar{\theta} E g_{I \bar{J}}(\Phi, \bar{\Phi}) g_{K \bar{L}}(\Phi, \bar{\Phi}) \mathcal{D}^{\alpha} \Phi^{I} \mathcal{D}_{\alpha} \Phi^{K} \overline{\mathcal{D}}_{\dot{\alpha}} \bar{\Phi}^{\bar{J}} \overline{\mathcal{D}}^{\dot{\alpha}} \bar{\Phi}^{\bar{L}}
$$

are independent super-Weyl invariants. In general, (7.5) should be added to (2.6), although it is not present in the $\mathcal{N}=2$ case, as follows from (2.20). Similarly, the super-Weyl 
anomaly (3.17) may include, in general, an additional contribution ${ }^{10}$

$$
\int \mathrm{d}^{4} x \mathrm{~d}^{2} \theta \mathrm{d}^{2} \bar{\theta} E(\sigma+\bar{\sigma}) g_{I \bar{J}}(\Phi, \bar{\Phi}) g_{K \bar{L}}(\Phi, \bar{\Phi}) \mathcal{D}^{\alpha} \Phi^{I} \mathcal{D}_{\alpha} \Phi^{K} \overline{\mathcal{D}}_{\dot{\alpha}} \bar{\Phi}^{\bar{J}} \overline{\mathcal{D}}^{\dot{\alpha}} \bar{\Phi}^{\bar{L}}
$$

It would be interesting to extend the analysis of the last three sections to the case of local $\mathcal{N}=2$ supersymmetry. Then the action (4.1) must be replaced with

$$
S[\mathbb{V} ; X, \bar{X}]=-\frac{\mathrm{i}}{8} \int \mathrm{d}^{4} x \mathrm{~d}^{4} \theta \mathcal{E} X(W(\mathbb{V}))^{2}+\text { c.c. }
$$

Here $X$ is a background chiral scalar superfield containing the dilaton and axion as the lowest component, and $W$ is the field strength of a vector multiplet. The latter is a reduced chiral superfield,

$$
\overline{\mathcal{D}}_{i}^{\dot{\alpha}} W=0, \quad\left(\mathcal{D}^{i j}+4 S^{i j}\right) W=\left(\overline{\mathcal{D}}^{i j}+4 \bar{S}^{i j}\right) \bar{W},
$$

see [35] for the technical details. There are three different realisations for the unconstrained prepotential $\mathbb{V}$ in (7.7). One option is to introduce a curved-superspace extension of Mezincescu's prepotential [67] (see also [68]), $V_{i j}=V_{j i}$, which is an unconstrained real SU(2) triplet. The expression for $W$ in terms of $V_{i j}$ was derived in [69], and is given by

$$
W=\frac{1}{4} \bar{\Delta}\left(\mathcal{D}^{i j}+4 S^{i j}\right) V_{i j},
$$

where $\bar{\Delta}$ is the chiral projection operator [36, 37]. Another option is the analytic prepotential $V^{++}$which originates within the harmonic superspace approach [70,71]. Finally, one can work with the tropical prepotential $V(\zeta)$ corresponding to the projective superspace approach [72-74]. It is not completely obvious which of the three prepotential is the best choice to perform loop calculations in supergravity.

\section{Acknowledgments}

I am grateful to Stefan Theisen and Arkady Tseytlin for discussions, and to Darren Grasso and Ian McArthur for comments on the manuscript. This work is supported in part by the Australian Research Council, project No. DP200101944.

\section{A Super-Weyl transformations}

The simplest approach to describe $\mathcal{N}=1$ conformal supergravity in superspace is to make use of the Grimm-Wess-Zumino geometry [30, 31], which is at the heart of the WessZumino formulation for old minimal supergravity [32], in conjunction with the super-Weyl transformations $[75,76]$. The geometry of curved superspace is described by covariant derivatives of the form

$$
\mathcal{D}_{A}=\left(\mathcal{D}_{a}, \mathcal{D}_{\alpha}, \overline{\mathcal{D}}^{\dot{\alpha}}\right)=E_{A}{ }^{M} \partial_{M}+\frac{1}{2} \Omega_{A}{ }^{b c} M_{b c},
$$

\footnotetext{
${ }^{10}$ The super-Weyl invariant (7.5) and anomaly contribution (7.6) were missed in the first and second arXiv versions of this paper. I thank Adam Schwimmer and Stefan Theisen for bringing the structures (7.5) and (7.6) to my attention.
} 
which obey the graded commutation relations [34]

$$
\begin{aligned}
\left\{\mathcal{D}_{\alpha}, \overline{\mathcal{D}}_{\dot{\alpha}}\right\} & =-2 \mathrm{i} \mathcal{D}_{\alpha \dot{\alpha}}, \\
\left\{\mathcal{D}_{\alpha}, \mathcal{D}_{\beta}\right\} & =-4 \bar{R} M_{\alpha \beta}, \quad\left\{\overline{\mathcal{D}}_{\dot{\alpha}}, \overline{\mathcal{D}}_{\dot{\beta}}\right\}=4 R \bar{M}_{\dot{\alpha} \dot{\beta}}, \\
{\left[\mathcal{D}_{\alpha}, \mathcal{D}_{\beta \dot{\beta}}\right] } & =\mathrm{i} \varepsilon_{\alpha \beta}\left(\bar{R} \overline{\mathcal{D}}_{\dot{\beta}}+G_{\dot{\beta}}^{\gamma} \mathcal{D}_{\gamma}-\mathcal{D}^{\gamma} G^{\delta}{ }_{\dot{\beta}} M_{\gamma \delta}+2 \bar{W}_{\dot{\beta}}{ }^{\dot{\gamma} \dot{\delta}} \bar{M}_{\dot{\gamma} \dot{\delta}}\right)+\mathrm{i} \overline{\mathcal{D}}_{\dot{\beta}} \bar{R} M_{\alpha \beta}, \\
{\left[\overline{\mathcal{D}}_{\dot{\alpha}}, \mathcal{D}_{\beta \dot{\beta}}\right] } & =-\mathrm{i} \varepsilon_{\dot{\alpha} \dot{\beta}}\left(R \mathcal{D}_{\beta}+G_{\beta}{ }^{\dot{\gamma}} \overline{\mathcal{D}}_{\dot{\gamma}}-\overline{\mathcal{D}}^{\dot{\gamma}} G_{\beta} \dot{\delta} \bar{M}_{\dot{\gamma} \dot{\delta}}+2 W_{\beta}{ }^{\gamma \delta} M_{\gamma \delta}\right)-\mathrm{i} \mathcal{D}_{\beta} R \bar{M}_{\dot{\alpha} \dot{\beta}} .
\end{aligned}
$$

Here the torsion tensors $R, G_{a}=\bar{G}_{a}$ and $W_{\alpha \beta \gamma}=W_{(\alpha \beta \gamma)}$ satisfy the Bianchi identities:

$$
\begin{aligned}
\overline{\mathcal{D}}_{\dot{\alpha}} R & =0, & & \overline{\mathcal{D}}_{\dot{\alpha}} W_{\alpha \beta \gamma}=0, \\
\overline{\mathcal{D}}^{\dot{\gamma}} G_{\alpha \dot{\gamma}} & =\mathcal{D}_{\alpha} R, & & \mathcal{D}^{\gamma} W_{\alpha \beta \gamma}=\mathrm{i} \mathcal{D}_{(\alpha}{ }^{\dot{\gamma}} G_{\beta) \dot{\gamma}} .
\end{aligned}
$$

The infinitesimal super-Weyl transformation is given by

$$
\begin{aligned}
\delta_{\sigma} \mathcal{D}_{\alpha} & =\left(\bar{\sigma}-\frac{1}{2} \sigma\right) \mathcal{D}_{\alpha}+\mathcal{D}^{\beta} \sigma M_{\alpha \beta}, \\
\delta_{\sigma} \overline{\mathcal{D}}_{\dot{\alpha}} & =\left(\sigma-\frac{1}{2} \bar{\sigma}\right) \overline{\mathcal{D}}_{\dot{\alpha}}+\left(\overline{\mathcal{D}}^{\dot{\beta}} \bar{\sigma}\right) \bar{M}_{\dot{\alpha} \dot{\beta}}, \\
\delta_{\sigma} \mathcal{D}_{\alpha \dot{\alpha}} & =\frac{1}{2}(\sigma+\bar{\sigma}) \mathcal{D}_{\alpha \dot{\alpha}}+\frac{\mathrm{i}}{2} \overline{\mathcal{D}}_{\dot{\alpha}} \bar{\sigma} \mathcal{D}_{\alpha}+\frac{\mathrm{i}}{2} \mathcal{D}_{\alpha} \sigma \overline{\mathcal{D}}_{\dot{\alpha}}+\mathcal{D}^{\beta}{ }_{\dot{\alpha}} \sigma M_{\alpha \beta}+\mathcal{D}_{\alpha} \dot{\beta} \bar{\sigma} \bar{M}_{\dot{\alpha} \dot{\beta}} .
\end{aligned}
$$

It generates the following transformations of the torsion superfields

$$
\begin{aligned}
\delta_{\sigma} R & =2 \sigma R+\frac{1}{4}\left(\overline{\mathcal{D}}^{2}-4 R\right) \bar{\sigma}, \\
\delta_{\sigma} G_{\alpha \dot{\alpha}} & =\frac{1}{2}(\sigma+\bar{\sigma}) G_{\alpha \dot{\alpha}}+\mathrm{i} \mathcal{D}_{\alpha \dot{\alpha}}(\sigma-\bar{\sigma}), \\
\delta_{\sigma} W_{\alpha \beta \gamma} & =\frac{3}{2} \sigma W_{\alpha \beta \gamma} .
\end{aligned}
$$

Here the super-Weyl parameter $\sigma$ is a covariantly chiral scalar superfield, $\overline{\mathcal{D}}_{\dot{\alpha}} \sigma=0$. The super-Weyl transformations belong to the gauge group of conformal supergravity.

Open Access. This article is distributed under the terms of the Creative Commons Attribution License (CC-BY 4.0), which permits any use, distribution and reproduction in any medium, provided the original author(s) and source are credited.

\section{References}

[1] M.K. Gaillard and B. Zumino, Duality Rotations for Interacting Fields, Nucl. Phys. B 193 (1981) 221 [INSPIRE].

[2] B. Zumino, Duality rotations, in Quantum Structure of Space and Time, M.J. Duff and C.J. Isham eds., Cambridge University Press (1982).

[3] S. Ferrara, J. Scherk and B. Zumino, Algebraic Properties of Extended Supergravity Theories, Nucl. Phys. B 121 (1977) 393 [InSPIRE]. 
[4] E. Cremmer and B. Julia, The $N=8$ Supergravity Theory. 1. The Lagrangian, Phys. Lett. $B$ 80 (1978) 48 [INSPIRE].

[5] E. Cremmer and B. Julia, The SO(8) supergravity, Nucl. Phys. B 159 (1979) 141 [InSPIRE].

[6] P. Aschieri, S. Ferrara and B. Zumino, Duality Rotations in Nonlinear Electrodynamics and in Extended Supergravity, Riv. Nuovo Cim. 31 (2008) 625 [arXiv:0807.4039] [INSPIRE].

[7] G.W. Gibbons and D.A. Rasheed, Electric-magnetic duality rotations in nonlinear electrodynamics, Nucl. Phys. B 454 (1995) 185 [hep-th/9506035] [INSPIRE].

[8] G.W. Gibbons and D.A. Rasheed, $\mathrm{SL}(2, \mathbb{R})$ invariance of nonlinear electrodynamics coupled to an axion and a dilaton, Phys. Lett. B 365 (1996) 46 [hep-th/9509141] [INSPIRE].

[9] M.K. Gaillard and B. Zumino, Selfduality in nonlinear electromagnetism, in Supersymmetry and Quantum Field Theory, J. Wess and V.P. Akulov eds., Springer Verlag (1998), pp. 121-129 [hep-th/9705226] [INSPIRE].

[10] M.K. Gaillard and B. Zumino, Nonlinear electromagnetic selfduality and Legendre transformations, in A Newton Institute Euroconference on Duality and Supersymmetric Theories, D.I. Olive and P.C. West eds., Cambridge University Press (1999), pp. 33-48 [hep-th/9712103] [INSPIRE].

[11] M. Araki and Y. Tanii, Duality symmetries in nonlinear gauge theories, Int. J. Mod. Phys. A 14 (1999) 1139 [hep-th/9808029] [INSPIRE].

[12] S.M. Kuzenko and S. Theisen, Nonlinear selfduality and supersymmetry, Fortsch. Phys. 49 (2001) 273 [hep-th/0007231] [INSPIRE].

[13] Y. Tanii, Introduction to Supergravity, Springer (2014).

[14] E.S. Fradkin and A.A. Tseytlin, Quantum Equivalence of Dual Field Theories, Annals Phys. 162 (1985) 31 [INSPIRE].

[15] R. Roiban and A.A. Tseytlin, On Duality Symmetry in Perturbative Quantum Theory, JHEP 10 (2012) 099 [arXiv: 1205.0176] [INSPIRE].

[16] I.L. Buchbinder, N.G. Pletnev and A.A. Tseytlin, "Induced" $N=4$ conformal supergravity, Phys. Lett. B 717 (2012) 274 [arXiv:1209.0416] [InSPIRE].

[17] F. Ciceri and B. Sahoo, Towards the full $N=4$ conformal supergravity action, JHEP 01 (2016) 059 [arXiv : 1510.04999] [INSPIRE].

[18] D. Butter, F. Ciceri, B. de Wit and B. Sahoo, Construction of all $N=4$ conformal supergravities, Phys. Rev. Lett. 118 (2017) 081602 [arXiv:1609.09083] [INSPIRE].

[19] H. Osborn, Local couplings and $\mathrm{SL}(2, \mathbb{R})$ invariance for gauge theories at one loop, Phys. Lett. B 561 (2003) 174 [hep-th/0302119] [INSPIRE].

[20] E.S. Fradkin and A.A. Tseytlin, Asymptotic freedom in extended conformal supergravities, Phys. Lett. B 110 (1982) 117 [INSPIRE].

[21] E.S. Fradkin and A.A. Tseytlin, One Loop $\beta$-function in Conformal Supergravities, Nucl. Phys. B 203 (1982) 157 [INSPIRE].

[22] S.M. Paneitz, A quartic conformally covariant differential operator for arbitrary pseudo-Riemannian manifolds, MIT preprint (1983), published posthumously in SIGMA 4 (2008) 036 [arXiv:0803.4331] [INSPIRE].

[23] R.J. Riegert, A Nonlocal Action for the Trace Anomaly, Phys. Lett. B 134 (1984) 56 [INSPIRE]. 
[24] D. Butter and S.M. Kuzenko, Nonlocal action for the super-Weyl anomalies: A new representation, JHEP 09 (2013) 067 [arXiv: 1307.1290] [INSPIRE].

[25] D. Butter, B. de Wit, S.M. Kuzenko and I. Lodato, New higher-derivative invariants in $N=2$ supergravity and the Gauss-Bonnet term, JHEP 12 (2013) 062 [arXiv:1307.6546] [INSPIRE].

[26] L.K. Hua, Harmonic Analysis of Functions of Several Complex Variables in the Classical Domains, American Mathematical Society, Providence U.S.A. (1963).

[27] M. Arai, S.M. Kuzenko and U. Lindström, HyperKähler $\sigma$-models on cotangent bundles of Hermitian symmetric spaces using projective superspace, JHEP 02 (2007) 100 [hep-th/0612174] [INSPIRE].

[28] J. Gomis, P.-S. Hsin, Z. Komargodski, A. Schwimmer, N. Seiberg and S. Theisen, Anomalies, Conformal Manifolds, and Spheres, JHEP 03 (2016) 022 [arXiv:1509.08511] [INSPIRE].

[29] A. Schwimmer and S. Theisen, Moduli Anomalies and Local Terms in the Operator Product Expansion, JHEP 07 (2018) 110 [arXiv:1805.04202] [INSPIRE].

[30] R. Grimm, J. Wess and B. Zumino, Consistency Checks on the Superspace Formulation of Supergravity, Phys. Lett. B 73 (1978) 415 [InSPIRE].

[31] R. Grimm, J. Wess and B. Zumino, A Complete Solution of the Bianchi Identities in Superspace, Nucl. Phys. B 152 (1979) 255 [INSPIRE].

[32] J. Wess and B. Zumino, Superfield Lagrangian for Supergravity, Phys. Lett. B 74 (1978) 51 [INSPIRE].

[33] J. Wess and J. Bagger, Supersymmetry and Supergravity, Princeton University Press (1992).

[34] I.L. Buchbinder and S.M. Kuzenko, Ideas and Methods of Supersymmetry and Supergravity or a Walk Through Superspace, IOP, Bristol U.K. (1998).

[35] S.M. Kuzenko, U. Lindström, M. Roček and G. Tartaglino-Mazzucchelli, $4 D N=2$ Supergravity and Projective Superspace, JHEP 09 (2008) 051 [arXiv:0805.4683] [INSPIRE].

[36] M. Müller, Consistent Classical Supergravity Theories, in Lecture Notes in Physics 336, Springer, Berlin Germany (1989).

[37] S.M. Kuzenko and G. Tartaglino-Mazzucchelli, Different representations for the action principle in $4 D N=2$ supergravity, JHEP 04 (2009) 007 [arXiv:0812.3464] [INSPIRE].

[38] I.L. Buchbinder, S.M. Kuzenko and A.A. Tseytlin, On low-energy effective actions in $N=2$, $N=4$ superconformal theories in four-dimensions, Phys. Rev. D 62 (2000) 045001 [hep-th/9911221] [INSPIRE].

[39] B. de Wit, S. Katmadas and M. van Zalk, New supersymmetric higher-derivative couplings: Full $N=2$ superspace does not count!, JHEP 01 (2011) 007 [arXiv:1010.2150] [INSPIRE].

[40] M. Henningson, Extended superspace, higher derivatives and $\mathrm{SL}(2, \mathbb{Z})$ duality, Nucl. Phys. B 458 (1996) 445 [hep-th/9507135] [INSPIRE].

[41] B. de Wit, M.T. Grisaru and M. Roček, Nonholomorphic corrections to the one loop $N=2$ superYang-Mills action, Phys. Lett. B 374 (1996) 297 [hep-th/9601115] [INSPIRE].

[42] S.M. Kuzenko, M. Ponds and E.S.N. Raptakis, New locally (super)conformal gauge models in Bach-flat backgrounds, arXiv:2005.08657 [INSPIRE].

[43] I.N. McArthur, Super-b $_{4}$ coefficients, Phys. Lett. B 128 (1983) 194 [InSPIRE]. 
[44] I.N. McArthur, Super-b ${ }_{4}$ Coefficients in Supergravity, Class. Quant. Grav. 1 (1984) 245 [INSPIRE].

[45] L. Bonora, P. Pasti and M. Tonin, Cohomologies and Anomalies in Supersymmetric Theories, Nucl. Phys. B 252 (1985) 458 [inSPIRE].

[46] I.L. Buchbinder and S.M. Kuzenko, Matter Superfields in External Supergravity: Green Functions, Effective Action and Superconformal Anomalies, Nucl. Phys. B 274 (1986) 653 [INSPIRE].

[47] I.L. Buchbinder and S.M. Kuzenko, Nonlocal Action for Supertrace Anomalies in Superspace of $N=1$ Supergravity, Phys. Lett. B 202 (1988) 233 [InSPIRE].

[48] A. Schwimmer and S. Theisen, Spontaneous Breaking of Conformal Invariance and Trace Anomaly Matching, Nucl. Phys. B 847 (2011) 590 [arXiv:1011.0696] [INSPIRE].

[49] S.M. Kuzenko, A. Schwimmer and S. Theisen, Comments on Anomalies in Supersymmetric Theories, J. Phys. A 53 (2020) 064003 [arXiv: 1909.07084] [InSPIRE].

[50] T. Levy, Y. Oz and A. Raviv-Moshe, $\mathcal{N}=1$ Liouville SCFT in Four Dimensions, JHEP 12 (2018) 122 [arXiv: 1810.02746] [inSPIRE].

[51] S.M. Kuzenko, Super-Weyl anomalies in $N=2$ supergravity and (non)local effective actions, JHEP 10 (2013) 151 [arXiv: 1307.7586] [INSPIRE].

[52] K. Nakagawa and Y. Nakayama, CP-violating super Weyl anomaly, Phys. Rev. D 101 (2020) 105013 [arXiv: 2002.01128] [INSPIRE].

[53] T. Levy, Y. Oz and A. Raviv-Moshe, $\mathcal{N}=2$ Liouville SCFT in four dimensions, JHEP 10 (2019) 006 [arXiv: 1907.08961] [InSPIRE].

[54] S. Cecotti and S. Ferrara, Supersymmetric Born-Infeld Lagrangians, Phys. Lett. B 187 (1987) 335 [INSPIRE].

[55] J. Bagger and A.S. Galperin, A New Goldstone multiplet for partially broken supersymmetry, Phys. Rev. D 55 (1997) 1091 [hep-th/9608177] [INSPIRE].

[56] D. Brace, B. Morariu and B. Zumino, Duality invariant Born-Infeld theory, in The Many Faces of the Superworld: Yury Golfand Memorial Volume, M. Shifman ed., World Scientific (2000), pp. 102-110 [hep-th/9905218] [INSPIRE].

[57] S.M. Kuzenko and S.A. McCarthy, Nonlinear selfduality and supergravity, JHEP 02 (2003) 038 [hep-th/0212039] [INSPIRE].

[58] V.P. Akulov and D.V. Volkov, Goldstone fields with spin 1/2, Theor. Math. Phys. 18 (1974) 28 [Teor. Mat. Fiz. 18 (1974) 39] [INSPIRE].

[59] M.T. Grisaru, N.K. Nielsen, W. Siegel and D. Zanon, Energy Momentum Tensors, Supercurrents, (Super)traces and Quantum Equivalence, Nucl. Phys. B 247 (1984) 157 [INSPIRE].

[60] I.L. Buchbinder and S.M. Kuzenko, Quantization of the classically equivalent theories in the superspace of simple supergravity and quantum equivalence, Nucl. Phys. B 308 (1988) 162 [INSPIRE].

[61] I.L. Buchbinder, S.M. Kuzenko and O.A. Solovev, One loop counterterms of Wess-Zumino model in the $N=1$ nonminimal supergravity background, Nucl. Phys. B 322 (1989) 277 [INSPIRE]. 
[62] D. Butter, One loop divergences and anomalies from chiral superfields in supergravity, arXiv:0911.5426 [INSPIRE].

[63] K.-H. Leung, Super heat kernel and one-loop divergence of super Yang-Mills theory in conformal supergravity, Prog. Theor. Exp. Phys. 2019 (2019) 103B02 [arXiv:1904.00105] [INSPIRE].

[64] K.-H. Leung, Super Heat Kernel of General Second Order Operators in $N=1$ Superspace and One-Loop Divergence of Dilaton-coupled SYM Theory, Phys. Rev. D 100 (2019) 055026 [arXiv: 1904.09746] [INSPIRE].

[65] I.N. McArthur, Superspace normal coordinates, Class. Quant. Grav. 1 (1984) 233 [INSPIRE].

[66] T.E. Clark, O. Piguet and K. Sibold, The Absence of Radiative Corrections to the Axial Current Anomaly in Supersymmetric QED, Nucl. Phys. B 159 (1979) 1 [INSPIRE].

[67] L. Mezincescu, On the superfield formulation of O(2) supersymmetry, JINR-P2-12572 (1979) [INSPIRE].

[68] P.S. Howe, K.S. Stelle and P.K. Townsend, Supercurrents, Nucl. Phys. B 192 (1981) 332 [INSPIRE].

[69] D. Butter and S.M. Kuzenko, New higher-derivative couplings in $4 D N=2$ supergravity, JHEP 03 (2011) 047 [arXiv: 1012.5153] [INSPIRE].

[70] A.S. Galperin, E. Ivanov, S.N. Kalitsyn, V.I. Ogievetsky and E.S. Sokatchev, Unconstrained $N=2$ Matter, Yang-Mills and Supergravity Theories in Harmonic Superspace, Class. Quant. Grav. 1 (1984) 469 [Corrigendum ibid. 2 (1985) 127] [INSPIRE].

[71] A.S. Galperin, E.A. Ivanov, V.I. Ogievetsky and E.S. Sokatchev, Harmonic Superspace, Cambridge University Press, Cambridge U.K. (2001).

[72] A. Karlhede, U. Lindström and M. Roček, Selfinteracting Tensor Multiplets in $N=2$ Superspace, Phys. Lett. B 147 (1984) 297 [INSPIRE].

[73] U. Lindström and M. Roček, New HyperKähler Metrics and New Supermultiplets, Commun. Math. Phys. 115 (1988) 21 [inSPIRE].

[74] U. Lindström and M. Roček, $N=2$ SuperYang-Mills Theory in Projective Superspace, Commun. Math. Phys. 128 (1990) 191 [INSPIRE].

[75] W. Siegel, Solution to Constraints in Wess-Zumino Supergravity Formalism, Nucl. Phys. B 142 (1978) 301 [INSPIRE].

[76] P.S. Howe and R.W. Tucker, Scale Invariance in Superspace, Phys. Lett. B 80 (1978) 138 [INSPIRE]. 